TRANSACTIONS OF THE

AMERICAN MATHEMATICAL SOCIETY

Volume 348, Number 2, February 1996

\title{
FACTORIZATIONS OF SIMPLE ALGEBRAIC GROUPS
}

\author{
MARTIN W. LIEBECK, JAN SAXL, AND GARY M. SEITZ
}

\begin{abstract}
We determine all factorizations of simple algebraic groups as the product of two maximal closed connected subgroups. Additional results are established which drop the maximality assumption, and applications are given to the study of subgroups of classical groups transitive on subspaces of a given dimension.
\end{abstract}

\section{INTRODUCTION}

Let $G$ be a simple algebraic group over an algebraically closed field $K$ of characteristic $p$ (allowing $p=0$ ). In this paper we determine all factorizations $G=X Y$ of $G$ as a product of two maximal closed subgroups $X$ and $Y$. Various cases of this problem have been studied by other authors. The "parabolic" factorizations - that is, the factorizations in which one of the factors $X, Y$ is a parabolic subgroupwere determined by A. Onishchik [On] (and also by I.L. Kantor [Ka] when $p=0$ ). We also mention that the maximal factorizations of the finite simple groups were determined in [LPS]. Our results form an interesting contrast with those of [LPS]; there are far fewer maximal factorizations of simple algebraic groups than there are of finite simple groups, but a few of those occurring in the algebraic case have no counterpart in the finite case.

One consequence of our results is the determination of all closed reductive subgroups of classical algebraic groups $G$ which act transitively on the set of totally singular or non-degenerate subspaces of some fixed dimension of the usual module for $G$.

We shall give complete proofs, including the parabolic cases covered by [On, Ka], since our methods are somewhat more straightforward than those of Onishchik and Kantor. In particular, we have the advantage of the substantial information on maximal subgroups of simple algebraic groups of exceptional type provided by [Se2].

We state our results separately for $G$ exceptional and $G$ classical. For $G$ exceptional, we give in fact all factorizations $G=X Y(X, Y$ closed), with no maximality assumptions on $X, Y$. It is elementary to see that if $G=X Y$, then also $G=X^{0} Y^{0}$ and $G=X^{g} Y^{h}$ for any $g, h \in G$ (Lemma 1.1); thus it is sufficient to list all possibilities for $X^{0}$ and $Y^{0}$ up to $G$-conjugacy.

To state our first result, we need to explain a little notation. In the root system of type $F_{4}$, the long roots form a $D_{4}$ subsystem, and the short roots a subsystem which we denote by $\tilde{D}_{4}$. When $p=2$, the corresponding root groups in $F_{4}$ generate subgroups $D_{4}, \tilde{D}_{4}$; these are contained in subgroups $B_{4}, C_{4}$, respectively. Similarly,

Received by the editors May 3, 1994 and, in revised form, January 30, 1995.

1991 Mathematics Subject Classification. Primary 20G15.

(C)1996 American Mathematical Society 
when $p=3$ and $G=G_{2}$, the long and short root subsystems give subgroups $A_{2}$ and $\tilde{A}_{2}$, respectively.

Theorem A. Let $G$ be a simple algebraic group of exceptional type in characteristic $p$, and suppose that $G=X Y$ with $X, Y$ closed proper subgroups of $G$. Then one of the following holds:

(i) $G=F_{4}, p=2$ and $X^{0}=D_{4}$ or $B_{4}, Y^{0}=\tilde{D}_{4}$ or $C_{4}$;

(ii) $G=G_{2}, p=3$ and $X^{0}=A_{2}, Y^{0}=\tilde{A}_{2}$.

Conversely, if $G, X, Y$ satisfy (i) or (ii), then $G=X Y$.

Remark. In (i), if $\tau$ is a graph automorphism of $F_{4}$, then $\tilde{D}_{4}=D_{4}^{\tau}$ and $C_{4}=B_{4}^{\tau}$.

To state our result for $G$ classical, we need some further notation. Let $V$ be the usual vector space associated with the classical group $G$; if $(G, p)=\left(B_{n}, 2\right)$, we take $V$ to be the associated $2 n$-dimensional symplectic space. Label the Dynkin diagram of $G$ as in [Bou, p. 250], and let $P_{i}$ be the parabolic subgroup of $G$ obtained by deleting the $i$ th node of the Dynkin diagram. Thus $P_{i}$ is the stabilizer in $G$ of a totally singular $i$-subspace of $V$ except when $(G, i)=\left(D_{n}, n-1\right.$ or $\left.n\right)$; in the exceptional case, $P_{n-1}$ and $P_{n}$ are stabilizers of totally singular $n$-subspaces in different $G$-orbits. When $G \neq S L_{n}$, let $N_{i}$ denote the connected stabilizer in $G$ of a non-degenerate subspace of $V$ of dimension $i$ with $i \leq \operatorname{dim} V / 2$; and when $(G, p)=\left(S O_{2 n}, 2\right)$, let $N_{1}$ denote the connected stabilizer of a nonsingular 1-space. Finally, if $\lambda$ is a dominant weight, denote by $V_{G}(\lambda)$ the rational irreducible $K G$ module with highest weight $\lambda$.

Theorem B. Let $G$ be a simple algebraic group of classical type in characteristic $p$ with (irreducible) natural module $V$, and suppose that $G=X Y$ with $X, Y$ maximal closed connected subgroups of $G$. Then $G=X Y$ is one of the following factorizations:

(1) parabolic factorizations:

$$
\begin{aligned}
& S L_{2 m}=S p_{2 m} P_{1}=S p_{2 m} P_{2 m-1}(m \geq 2), \\
& S O_{2 m}=N_{1} P_{m}=N_{1} P_{m-1}(m \geq 4), \\
& S O_{8}=B_{3} P_{i}(i=1,3,4)\left(\text { where } V \downarrow B_{3}=V_{B_{3}}\left(\lambda_{3}\right)\right), \\
& S O_{7}=G_{2} P_{1}, \\
& S p_{6}=G_{2} P_{1}(p=2) ;
\end{aligned}
$$

(2) non-parabolic factorizations, $p$ arbitrary:

$$
\begin{aligned}
S O_{2 m}= & \left(S p_{m} \otimes S p_{2}\right) N_{1}(m \text { even }) \\
S O_{16}= & B_{4} N_{1}\left(\text { where } V \downarrow B_{4}=V_{B_{4}}\left(\lambda_{4}\right)\right), \\
P S O_{8}= & B_{3} B_{3}^{\tau}=B_{3} N_{3}=B_{3}\left(S p_{4} \otimes S p_{2}\right) \text { (where } \\
& \left.V \downarrow B_{3}=V_{B_{3}}\left(\lambda_{3}\right) \text { and } \tau \text { is a triality aut. of } P S O_{8}\right), \\
S O_{7}= & G_{2} N_{1} ;
\end{aligned}
$$

(3) non-parabolic factorizations, $p=3$ :

$$
\begin{aligned}
& S O_{25}=F_{4} N_{1} \quad\left(\text { where } V \downarrow F_{4}=V_{F_{4}}\left(\lambda_{4}\right)\right), \\
& S O_{13}=C_{3} N_{1} \quad\left(\text { where } V \downarrow C_{3}=V_{C_{3}}\left(\lambda_{2}\right)\right) ;
\end{aligned}
$$


(4) non-parabolic factorizations, $p=2$ :

$$
\begin{aligned}
& S p_{2 m}=S O_{2 m} N_{2 k}(1 \leq k \leq m-1), \\
& S O_{56}=E_{7} N_{1}\left(\text { where } V \downarrow E_{7}=V_{E_{7}}\left(\lambda_{7}\right)\right), \\
& S O_{32}=D_{6} N_{1}\left(\text { where } V \downarrow D_{6}=V_{D_{6}}\left(\lambda_{5}\right) \text { or } V_{D_{6}}\left(\lambda_{6}\right)\right), \\
& S O_{20}=A_{5} N_{1}\left(\text { where } V \downarrow A_{5}=V_{A_{5}}\left(\lambda_{3}\right)\right), \\
& S p_{6}=G_{2} N_{2}=G_{2} S O_{6} .
\end{aligned}
$$

Remarks. (1) Note that factorizations of $S p_{2 m}$ with $p=2$ give corresponding factorizations of $S O_{2 m+1}$, via a surjective morphism from one group to the other (the latter factorizations are not listed in Theorem B).

(2) It is possible to drop the maximality assumptions on $X$ and $Y$ in Theorem B, and to determine all factorizations $G=X Y$ where $X, Y$ are closed proper subgroups and each is either reductive or parabolic. We do this in an Appendix at the end of the paper.

Corollary 1. If $G$ is classical with natural module $V$, and $G=X Y$ with $X, Y$ closed connected proper subgroups, then there is an automorphism $\alpha$ of $G$ (as abstract group) such that $X^{\alpha}$ or $Y^{\alpha}$ is reducible on $V$.

Theorem B, together with Theorem $\mathrm{C}$ in the Appendix, determines all closed reductive subgroups of classical groups which act transitively on the set of totally singular or non-degenerate subspaces of some fixed dimension. In the next corollary, we highlight one particular case.

Corollary 2. Let $V$ be a vector space of dimension $n$ over an algebraically closed field of characteristic p. Suppose $G$ is a closed proper subgroup of $S L(V)$ which acts transitively on the set of $i$-dimensional subspaces of $V$ for some $i<n$. Then either $G=S p(V)$ with $n$ even and $i=1$ or $n-1$, or $G=G_{2}$ with $n=6, p=2$ and $i=1$ or 5 .

This can be deduced from Theorems A and B as follows. Let $G$ be as in the hypothesis of Corollary 2. Then $S L(V)=G P_{i}$. By Lemma 1.1 we may take $G$ to be connected. From Theorem B we deduce that $i=1$ or $n-1$ and $G \leq S p(V)$. If $G<S p(V)$, then $S p(V)=G P_{1}$, whence Theorem B gives $n=6, p=2$ and $G \leq G_{2}$; then $G_{2}=G P_{1}$, so $G=G_{2}$ by Theorem A.

The layout of the rest of the paper is as follows. In section 1 , we demonstrate the existence of all the factorizations in Theorems A and B. Section 2 contains some general lemmas on factorizations, and in section 3 we prove Theorem A. Theorem B is established in sections 4 and 5: section 4 classifies the parabolic factorizations of classical groups, and section 5 the non-parabolic factorizations. Finally, in the Appendix we show how the maximality assumptions of Theorem B can be relaxed, determining all factorizations $G=X Y$ with $X, Y$ reductive or parabolic.

Acknowledgment. The first and third authors acknowledge the support of a NATO Collaborative Research Grant.

\section{Existence of the factorizations in Theorems A and B}

As before, let $G$ be a simple algebraic group over an algebraically closed field $K$ of characteristic $p$. In this section we establish the existence of the factorizations in Theorems A and B. We thank Professor R. Steinberg for suggesting the method used in Proposition 1.9 below, which is conceptually more natural than our original proof. 
Lemma 1.1. Suppose that $G=X Y$ with $X, Y$ proper closed subgroups. Then

(i) $G=X^{0} Y^{0}$,

(ii) $G=X^{g} Y^{h}$ for any $g, h \in G$,

(iii) if $X \leq N_{G}(D)$ for some non-central subgroup $D$ of $G$, then $Y$ contains no conjugate of $D$.

Proof. To prove (ii), let $g=x y$ with $x \in X, y \in Y$. Then $G=(X Y)^{y}=X^{x y} Y=$ $X^{g} Y$. Repeating the argument gives $G=X^{g} Y^{h}$. For (iii), note that $Y$ is transitive on the conjugates of $D$, so cannot contain one of these.

We now prove (i). Since $X$ is closed, $G / X$ is an irreducible variety. The group $Y$ acts transitively on $G / X$, so $Y^{0}$ has finitely many orbits, say $\Delta_{1}, \ldots, \Delta_{k}$, and these are permuted transitively by $Y / Y^{0}$. Suppose that $k>1$. As $G / X$ is irreducible, the orbits $\Delta_{i}$ are not closed. Some orbit, hence every orbit, is open in its closure, hence is open and dense in $G / X$. But this means that the complement of $\Delta_{1}$, namely $\Delta_{2} \cup \ldots \cup \Delta_{k}$, is open and closed, hence is equal to $G / X$, a contradiction. Therefore $k=1$, and $Y^{0}$ is transitive on $G / X$. In other words, $G=X Y^{0}$. Repeating the argument, we see that $G=X^{0} Y^{0}$.

In the first three propositions we give elementary geometric proofs of some of the factorizations in Theorem B.

Proposition 1.2. The parabolic factorizations in Theorem B(1) occur.

Proof. The factorizations $S L_{2 m}=S p_{2 m} P_{1}=S p_{2 m} P_{2 m-1}$ are clear, as $S p_{2 m}$ is transitive on the sets of 1 -spaces and hyperplanes in $2 m$-dimensional space.

Next we show that $S O_{2 m}=N_{1} P_{m}=N_{1} P_{m-1}$. Let $V$ be the natural $2 m$ dimensional module for $G=S O_{2 m}$. Pick a Levi subgroup $L=G L_{m}$ of $G$. Then $L$ fixes a pair $E, F$ of totally singular $m$-spaces with $V=E \oplus F$, and every nonsingular 1-space contains a vector $e+f$ such that $e \in E, f \in F$ and $(e, f)=1$.

Fix $e_{0} \in E$ with $e_{0} \neq 0$. The stabilizer $L_{e_{0}}$ acts transitively on vectors $f \in F$ such that $\left(e_{0}, f\right)=1$ (indeed, so does $\left.\left(L^{\prime}\right)_{e_{0}}\right)$. Since $L^{\prime}$ is transitive on the nonzero vectors in $E$, it follows that $L^{\prime}$ is transitive on the set of all nonsingular 1-spaces in $V$. Hence $G=N_{1} L^{\prime}$. Since conjugates of $L^{\prime}$ lie in $P_{m}$ and in $P_{m-1}$, it follows that $G=N_{1} P_{m}=N_{1} P_{m-1}$.

In particular, $S O_{8}=N_{1} P_{3}=N_{1} P_{4}$. The image of $N_{1}$ under a triality automorphism of $D_{4}$ is an irreducible $B_{3}$; hence $S O_{8}=B_{3} P_{i}$ for $i=1,3,4$.

It remains to show that $S O_{7}=G_{2} P_{1}$ and $S p_{6}=G_{2} P_{1}(p=2)$. The latter follows from the former on application of a surjective morphism $S O_{7} \rightarrow S p_{6}(p=2)$; so we need only prove that $S O_{7}=G_{2} P_{1}$. For this, it will suffice to show that $S O_{7}=G_{2} S O_{5}$, since $S O_{5}$ is a Levi subgroup of $P_{1}$. By the third paragraph, we know that $S_{8}=N_{1} S L_{4}$. Application of triality gives $S_{8}=B_{3} S O_{6}$. It follows that $N_{1}=\left(B_{3} \cap N_{1}\right) S O_{6}=G_{2} S O_{6}$; that is, $S O_{7}=G_{2} S O_{6}$. Again by the third paragraph, $S O_{6}=S O_{5} S L_{3}$. Inside $S O_{7}$, this $S L_{3}$ lies in $G_{2}$, so $S O_{7}=G_{2} S O_{5}$. Hence $\mathrm{SO}_{7}=G_{2} P_{1}$, as required.

Proposition 1.3. The factorizations in Theorem $B(2)$ (excluding $S O_{16}=B_{4} N_{1}$ ) all occur.

Proof. We established in the proof of 1.2 that $S O_{2 m}=N_{1} S L_{m}$. Let $V$ be the natural $2 m$-dimensional orthogonal module, and let $V=E \oplus F$ as in the proof of 1.2. Suppose that $m$ is even, and choose a subgroup $S$ of $S L_{m}$ with $S \cong S p_{m}$. For $0 \neq e \in E, S_{e}$ fixes a nonzero vector $d \in F$ with $(e, d)=0$, and is transitive 
on the set of vectors $f \in F$ with $(e, f)=1$. Since $S$ is transitive on the nonzero vectors in $E$, it follows that $S$ is transitive on the nonsingular 1-spaces in $V$, and so $S O_{2 m}=N_{1} S p_{m}=N_{1}\left(S p_{m} \otimes S p_{2}\right)$. This is the first factorization in Theorem $\mathrm{B}(2)$.

We showed in the proof of 1.2 that $S O_{7}=G_{2} S_{5}$, so $S O_{7}=G_{2} N_{1}$. And we also proved that $S O_{8}=B_{3} N_{1}$, so $P_{S O_{8}}=B_{3} B_{3}^{\tau}$ for any triality automorphism $\tau$ of $\mathrm{PSO}_{8}$.

Proposition 1.4. The following factorizations in Theorem B(4) occur:

$$
\begin{aligned}
& S p_{2 m}=S O_{2 m} N_{2 k}(p=2,1 \leq k \leq m-1), \\
& S p_{6}=G_{2} N_{2}(p=2), \\
& S p_{6}=G_{2} S O_{6}(p=2) .
\end{aligned}
$$

Proof. The first factorization is an immediate consequence of Witt's Lemma.

We showed in the proof of 1.2 that $S O_{7}=G_{2} N_{1}=G_{2} S O_{5}$. For $p=2$, application of a surjective morphism $S O_{7} \rightarrow S p_{6}$ yields $S p_{6}=G_{2} S O_{6}=G_{2} S p_{4}$. Therefore $S p_{6}=G_{2} S O_{6}=G_{2} N_{2}$.

The remaining factorizations in Theorems A and B are less easy to establish, and we use a method suggested by R. Steinberg.

Lemma 1.5. Let $U$ be a connected unipotent algebraic group over $K$, and suppose that $A, B$ are closed subgroups of $U$ such that $\operatorname{dim} A+\operatorname{dim} B-\operatorname{dim} A \cap B \geq \operatorname{dim} U$. Then $U=A B$.

Proof. Induct on $\operatorname{dim} U$. We may assume $B$ is proper and embed it in a maximal closed subgroup $M$ of $U$. If $A \leq M$ then, by induction, $M=A B$ and $\operatorname{dim} A+$ $\operatorname{dim} B-\operatorname{dim} A \cap B=\operatorname{dim} M$, contradicting the hypothesis. Hence $A \not \leq M$; so $U=A M$ and $\operatorname{dim} A \cap M=\operatorname{dim} A-1$.

Now the hypothesis gives $\operatorname{dim} A \cap M+\operatorname{dim} B-\operatorname{dim} A \cap M \cap B=\operatorname{dim} A-1+$ $\operatorname{dim} B-\operatorname{dim} A \cap B \geq \operatorname{dim} U-1=\operatorname{dim} M$. So by induction, $M=(A \cap M)$. B. Multiplying by $A$ we have the assertion.

Corollary 1.6. Let $B$ be a connected solvable algebraic group over $K$, and $X, Y$ closed subgroups with $\operatorname{dim} X+\operatorname{dim} Y-\operatorname{dim} X \cap Y \geq \operatorname{dim} B$. Then $B=X Y$.

Proof. We may assume $X$ and $Y$ are connected. Replace $X$ by a conjugate, if necessary, so that $X$ and $Y$ have tori, $T_{X}, T_{Y}$, each contained in a fixed maximal torus $T$ of $B$. In fact, we may also assume that $T_{X} \cap T_{Y}$ is a maximal torus of $X \cap Y$.

First we claim that $T_{X} T_{Y}$ is a closed subgroup of $T$. Indeed, the map $T \rightarrow T / T_{Y}$ is a morphism, so $T_{X}$ has closed image. Taking preimages we have the claim.

Next note that $R_{u}(X), R_{u}(Y)$ are contained in $R_{u}(B)$. Now $X \cap Y=$ $R_{u}(X \cap Y) .\left(T_{X} \cap T_{Y}\right)$. A dimension count shows that we have the hypotheses of Lemma 1.5, so $R_{u}(B)=R_{u}(X) \cdot R_{u}(Y)$.

Now consider $B / R_{u}(B)$, a torus. The images of $X$ and $Y$ are both subtori, so the argument of the second paragraph shows that the product of the images is a closed subgroup. Hence the preimage, $X R_{u}(B) Y=X Y$, is also a closed subgroup. Finally, the hypothesis forces $X Y=B$.

Lemma 1.7 ([St2, Lemma 2, p. 68]). Let $J$ be an algebraic group over $K$ acting algebraically on an algebraic variety $V$ over $K$, and let $H$ be a closed subgroup of $J$ with $J / H$ complete. Suppose that $U$ is a closed, $H$-invariant subset of $V$. Then J.U is closed. 
Proof. Although this is in [St2] we give a proof for completeness. Let $S=\{(x H, v)$ : $\left.x^{-1} v \in U\right\}$. Since $S$ is $H$-invariant, it is a well-defined subset of $J / H \times V$.

We claim $S$ is closed. Map $J \times V \rightarrow V$ via $(g, v) \rightarrow g^{-1} v$. Let $D$ be the preimage of $U$. Then $D$ is closed. The natural map $J \times V \rightarrow J / H \times V$ is an open map (see $[\mathrm{Hu}$, p. 86, Ex.4]). Under this map, $D$ and its complement have disjoint images. The assertion follows.

By hypothesis, $J / H$ is complete, so projecting $S$ to the second coordinate, we find that the image, J.U, is closed.

Corollary 1.8. Let $X, Y$ be closed subgroups of $G$ such that $\operatorname{dim} X+\operatorname{dim} Y-$ $\operatorname{dim} X \cap Y=\operatorname{dim} G$, and suppose that there are Borel subgroups $B_{X}, B_{Y}$ of $X, Y$ such that $B_{X} B_{Y}$ is closed. Then $G=X Y$.

Proof. Apply Lemma 1.7 to $J=X \times Y$ acting on $V=G$ with $(x, y)$ sending $g \rightarrow$ $x^{-1} g y(x \in X, y \in Y, g \in G)$, with $H=B_{X} \times B_{Y}$ and $U=B_{X} B_{Y}$. We conclude that $J . U=X Y$ is closed. Hence by the assumption on dimensions, $G=X Y$.

We are now in a position to establish the remaining factorizations in Theorems A and B.

Proposition 1.9. The following factorizations $G=X Y$ occur:

$$
\begin{aligned}
& F_{4}=D_{4} \tilde{D}_{4}(p=2)(\text { as in Theorem } \mathrm{A}), \\
& G_{2}=A_{2} \tilde{A}_{2}(p=3)(\text { as in Theorem } \mathrm{A}), \\
& \left.S O_{56}=E_{7} N_{1}(p=2) \text { (as in Theorem } \mathrm{B}(4)\right), \\
& S O_{32}=D_{6} N_{1}(p=2)(\text { as in Theorem } \mathrm{B}(4)), \\
& S O_{25}=F_{4} N_{1}(p=3)(\text { as in Theorem } \mathrm{B}(3)), \\
& \left.S O_{20}=A_{5} N_{1}(p=2) \text { (as in Theorem } \mathrm{B}(4)\right), \\
& S O_{16}=B_{4} N_{1}(\text { as in Theorem } \mathrm{B}(2)), \\
& \left.S O_{13}=C_{3} N_{1}(p=3) \text { (as in Theorem } \mathrm{B}(3)\right) .
\end{aligned}
$$

Proof. The embeddings $E_{7}<S O_{56}(p=2), D_{6}<S O_{32}(p=2), F_{4}<S O_{25}(p=3)$, $A_{5}<S O_{20}(p=2), B_{4}<S O_{16}$ and $C_{3}<S O_{13}(p=3)$, via the modules given in Theorem B, are well known (see 2.7 in section 2, for example, for some of them).

Let $G, X, Y$ be as in the statement of the proposition. We claim that we can choose $X$ and $Y$ such that

(a) there is a Borel subgroup $B_{G}$ of $G$ which contains Borel subgroups $B_{X}, B_{Y}$ of $X, Y$, respectively, and

(b) $(X \cap Y)^{0}$ is as follows (where $T_{i}$ denotes a rank $i$ torus):

\begin{tabular}{lccll}
\hline & $G$ & $X$ & $Y$ & $(X \cap Y)^{0}$ \\
\hline$(1)$ & $F_{4}$ & $D_{4}$ & $\tilde{D}_{4}$ & $T_{4}$ \\
$(2)$ & $G_{2}$ & $A_{2}$ & $\tilde{A}_{2}$ & $T_{2}$ \\
$(3)$ & $S O_{56}$ & $E_{7}$ & $N_{1}$ & $E_{6}$ \\
$(4)$ & $S O_{32}$ & $D_{6}$ & $N_{1}$ & $A_{5}$ \\
$(5)$ & $S O_{25}$ & $F_{4}$ & $N_{1}$ & $D_{4}$ \\
$(6)$ & $S O_{20}$ & $A_{5}$ & $N_{1}$ & $A_{2} A_{2}$ \\
$(7)$ & $S O_{16}$ & $B_{4}$ & $N_{1}$ & $B_{3}$ \\
$(8)$ & $S O_{13}$ & $C_{3}$ & $N_{1}$ & $A_{1} A_{1} A_{1}$ \\
\hline
\end{tabular}

In cases (1) and (2) this is easy: choose $X, Y$ to satisfy (a) and such that $X \cap Y$ contains a maximal torus $T$ of $G$. Then $(X \cap Y)^{0}$ is generated by $T$ together with any $T$-root subgroups lying in $X \cap Y$; but the root subgroups in $X$ correspond to 
long roots, whereas those in $Y$ correspond to short roots, and hence $(X \cap Y)^{0}=T$, as claimed.

The remaining cases (3)-(8) are similar to each other. In each case we claim first that we can find a closed connected subgroup $D$ of $X$ as in the last column of the above table, generated by long root subgroups of $X$, such that $V \downarrow D$ is as follows:

(3) $V \downarrow D=V_{E_{6}}\left(\lambda_{1}\right) \oplus V_{E_{6}}\left(\lambda_{6}\right) \oplus V_{2}$,

(4) $V \downarrow D=V_{A_{5}}\left(\lambda_{2}\right) \oplus V_{A_{5}}\left(\lambda_{4}\right) \oplus V_{2}$,

(5) $V \downarrow D=V_{D_{4}}\left(\lambda_{1}\right) \oplus V_{D_{4}}\left(\lambda_{3}\right) \oplus V_{D_{4}}\left(\lambda_{4}\right) \oplus V_{1}$,

(6) $V \downarrow D=\left(V_{A_{2}}\left(\lambda_{1}\right) \otimes V_{A_{2}}\left(\lambda_{2}\right)\right) \oplus\left(V_{A_{2}}\left(\lambda_{2}\right) \otimes V_{A_{2}}\left(\lambda_{1}\right)\right) \oplus V_{2}$,

(7) $V \downarrow D=V_{B_{3}}\left(\lambda_{1}\right) \oplus V_{B_{3}}\left(\lambda_{3}\right) \oplus V_{1}$,

(8) $\quad V \downarrow D=\left(V_{A_{1}}\left(\lambda_{1}\right) \otimes V_{A_{1}}\left(\lambda_{1}\right) \otimes V_{A_{1}}(0)\right) \oplus\left(V_{A_{1}}\left(\lambda_{1}\right) \otimes V_{A_{1}}(0) \otimes V_{A_{1}}\left(\lambda_{1}\right)\right)$ $\oplus\left(V_{A_{1}}(0) \otimes V_{A_{1}}\left(\lambda_{1}\right) \otimes V_{A_{1}}\left(\lambda_{1}\right)\right) \oplus V_{1}$

where $V_{i}(i=1$ or 2$)$ denotes a trivial submodule of dimension $i$. This claim is well known in cases (3), (4) and (5) - see for example [LS2, §2]. The claim is clear in case (6), since in this case $V \downarrow X$ is the wedge-cube of the usual 6-dimensional $X$-module; similarly in case (8), $V \downarrow X$ is a section of the wedge-square of the usual 6 -dimensional module. Finally for (7), take a subgroup $D_{4}$ of $X=B_{4}$ such that the spin module $V=V_{X}\left(\lambda_{4}\right)$ restricts to $D_{4}$ as $V_{D_{4}}\left(\lambda_{3}\right) \oplus V_{D_{4}}\left(\lambda_{4}\right)$. Now choose a subgroup $D=B_{3}$ of this $D_{4}$ fixing a nonsingular 1-space in one of the summands.

Thus $V \downarrow D$ is as above. Let $T_{X}$ be a maximal torus of $X$ and choose a basis $\mathcal{B}$ of $T_{X}$-weight vectors for $V$. All $T_{X}$-weight spaces have dimension 1 ; for a weight $\mu$, let $e_{\mu}$ be the chosen weight vector. In cases (3), (4) and (6), if $\lambda$ denotes the highest weight, we may take $V_{2}=\left\langle e_{\lambda}, e_{-\lambda}\right\rangle$, and we define $v=e_{\lambda}+e_{-\lambda}$; in the other cases we choose $v$ so that $V_{1}=\langle v\rangle$. Then $X_{v}^{0}=D$. Taking $Y=G_{v}^{0}$, we therefore have $(X \cap Y)^{0}=D$, establishing conclusion (b) above.

Now we argue that (a) holds. Let $B_{X}$ be a Borel subgroup of $X$ containing $T_{X}$. In cases (5) and (8) we may take $V_{1}=\langle v\rangle$ to be the 0 -weight space for $T_{X}$. Then $B_{X}$ fixes a complete flag $\mathcal{F}$ of $V$ determined by an ordered basis of the form $e_{\mu_{1}}, \ldots, e_{\mu_{m}}, v, e_{-\mu_{m}}, \ldots, e_{-\mu_{1}}$ (where $\left.\operatorname{dim} V=2 m+1\right)$. Then $Y_{\mathcal{F}}=\left(G_{v}\right)_{\mathcal{F}}^{0}$ is a Borel subgroup of $Y$. Hence the Borel subgroup $G_{\mathcal{F}}$ of $G$ contains Borel subgroups of $X$ and of $Y$, establishing (a) for these cases. Now consider cases (3), (4), (6). Here $B_{X}$ fixes a complete flag $\mathcal{F}$ of $V$ determined by an ordered basis of the form $e_{\mu_{1}}, \ldots, e_{\mu_{m}}, e_{-\mu_{m}}, \ldots, e_{-\mu_{1}}$. Since the Weyl group $W(X)$ is transitive on the set of weights appearing, the 2-space $\left\langle e_{\mu_{m}}, e_{-\mu_{m}}\right\rangle$ is fixed by a $W(X)$-conjugate of $D$; replace $V_{2}$ by this 2 -space and $v$ by the vector $e_{\mu_{m}}+e_{-\mu_{m}}$. Then $Y_{\mathcal{F}}=\left(G_{v}\right)_{\mathcal{F}}^{0}$ is again a Borel subgroup of $Y$, and (a) follows as before. Finally in case (7), a maximal torus of $D=B_{3}$ has 0 -weight space of dimension 2, which we may take to be $\left\langle e_{\mu_{m}}, e_{-\mu_{m}}\right\rangle$ in the previous argument. This argument now yields (a) for this case.

Thus (a) and (b) hold in all cases. Observe now that for all the cases (1)-(8) listed above, we have

$$
\operatorname{dim} X+\operatorname{dim} Y-\operatorname{dim} X \cap Y=\operatorname{dim} G
$$

and also

$$
\operatorname{dim} B_{X}+\operatorname{dim} B_{Y}-\operatorname{dim} B_{X \cap Y}=\operatorname{dim} B_{G} .
$$

Hence by Corollary 1.6 we have $B_{G}=B_{X} B_{Y}$. It now follows from Corollary 1.8 that $G=X Y$, as required. 
We have now established all the factorizations in Theorems A and B.

\section{Preliminaries for proofs of Theorems A And B}

Continue to assume that $G$ is a simple algebraic group over an algebraically closed field of characteristic $p$. The first two results of this section give useful information concerning parabolic factorizations of $G$.

Lemma 2.1. Suppose that $G=X Y$ with $X$ a proper closed subgroup, and $Y$ a proper parabolic subgroup of $G$. Then

(i) $X$ is reductive;

(ii) $X \cap Y$ is a parabolic subgroup of $X$;

(iii) $R_{u}(Y)$ and $R_{u}(X \cap Y)$ have equal dimensions (equal to $\operatorname{dim}(G / Y)$ );

(iv) $\operatorname{dim} X \geq 2 \operatorname{dim}(G / Y)+\operatorname{rank}(X)$;

(v) $X$ lies in no proper subgroup of maximal rank in $G$.

Proof. (i) Suppose that $X$ is not reductive, and let $U=R_{u}(X) \neq 1$. Then $X \leq$ $N_{G}(U)$. But the parabolic subgroup $Y$ contains a conjugate of $U$, so this contradicts 1.1(iii).

(ii) Let $Y=Q L$, where $Q=R_{u}(Y)$ and $L$ is a Levi subgroup of $Y$. Choose maximal tori $T_{X}, T$ lying in Borel subgroups $B_{X}, B$ of $X, G$ respectively, such that $T_{X} \leq T \leq L$ and $B_{X} \leq B \leq Y$. Then $X \cap Y$ contains $B_{X}$, hence is a parabolic subgroup of $X$.

(iii) We have $\operatorname{dim}(G / Y)=\operatorname{dim} R_{u}(Y)$; and by (ii),

$$
\operatorname{dim}(X / X \cap Y)=\operatorname{dim}\left(R_{u}(X \cap Y)\right) .
$$

Since $G=X Y, \operatorname{dim}(G / Y)=\operatorname{dim}(X / X \cap Y)$, and (iii) follows.

(iv) We have

$$
\begin{aligned}
\operatorname{dim} X & =\operatorname{dim}(G / Y)+\operatorname{dim}(X \cap Y) \geq \operatorname{dim}(G / Y)+\operatorname{dim} R_{u}(X \cap Y)+\operatorname{rank}(X) \\
& =2 \operatorname{dim}(G / Y)+\operatorname{rank}(X),
\end{aligned}
$$

using (iii) for the last equality.

(v) By way of contradiction, assume that $X$ is a proper subgroup of maximal rank in $G$. Then $T_{X}=T$. Write $X \cap Y=Q_{X} L_{X}$, where $Q_{X}$ is the unipotent radical and $L_{X}$ a Levi subgroup of $X \cap Y$. Since $T_{X}=T$, every root subgroup of $X$ is a root subgroup of $G$.

Suppose that $Q_{X} \not \leq Q$, where $Y=Q L$ as before, and pick a $T$-root subgroup $U_{\beta}$ lying in $Q_{X}$ but not in $Q$. Since every $T$-root subgroup of $Y=Q L$ lies in $Q$ or in $L$, we have $U_{\beta} \leq L$. This implies that $U_{-\beta} \leq L$. As $U_{-\beta} \leq X$ also, this gives $U_{-\beta} \leq L \cap X \leq Q_{X} L_{X}$, which is impossible as $U_{\beta} \leq Q_{X}$. Therefore $Q_{X} \leq Q$, and so $Q_{X}=Q$ by (iii). But then

$$
G=\left\langle Q^{G}\right\rangle=\left\langle Q^{Y X}\right\rangle=\left\langle Q^{X}\right\rangle \leq X,
$$

which is a contradiction. This completes the proof.

Lemma 2.2. Suppose that $G=X Y$ with $X$ a closed subgroup and $Y=Q L$ parabolic, with unipotent radical $Q$ and Levi subgroup L. Assume that the subsystem of the root system of $G$ corresponding to $L$ is fixed by $w_{0}$, the longest element of the Weyl group of $G$. Then $G=X L$. 
Proof. Choose maximal tori $T_{X}, T$ contained in Borel subgroups $B_{X}, B$ of $X, G$ respectively, with $T_{X} \leq T \leq L$ and $B_{X} \leq B \leq Y$. As in the proof of 2.1, write $X \cap Y=Q_{X} L_{X}$.

We first establish that $Q_{X} \cap L=1$. By our hypothesis concerning $w_{0}$, we can find a dominant weight $\lambda$ such that $V=V_{G}(\lambda)$ is self-dual and $G_{\left\langle v^{+}\right\rangle}=Y$ for some maximal vector $v^{+} \in V$. Let $\bar{w}_{0}$ be an element in the coset of $w_{0}$, and put $v^{-}=v^{+} \bar{w}_{0}$. Then $G_{\left\langle v^{+}\right\rangle,\left\langle v^{-}\right\rangle}=Y \cap Y^{\bar{w}_{0}}=L$.

Let $\delta=\lambda \downarrow T_{X}$, so that $v^{+}, v^{-}$afford the weights $\delta,-\delta$ for $T_{X}$, respectively. As $B_{X} \leq B, v^{+}$is a maximal vector for $B_{X}$ and $V \downarrow X$ has a composition factor of high weight $\delta$. Because $V$ is finite-dimensional, it follows that $\delta$ is a dominant weight. Since $G=X Y$, we have

$$
V=\left\langle v^{+} G\right\rangle=\left\langle v^{+} X\right\rangle .
$$

Consequently $V \downarrow X$ is an image of the Weyl module $W_{X}(\delta)$ in which the weights $\delta,-\delta$ appear with multiplicity 1 . Now $X_{\left\langle v^{+}\right\rangle}=X \cap Y=Q_{X} L_{X}$, so $X_{\left\langle v^{-}\right\rangle}=$ $\left(Q_{X} L_{X}\right)^{t}$, where $t=\bar{w}_{0}(X)$. As $L_{X}$ fixes $\left\langle v^{-}\right\rangle$, we have $L_{X}^{t}=L_{X}$ and $Q_{X}^{t}$ is the unipotent radical of the parabolic of $X$ opposite to $Q_{X} L_{X}$. Hence we conclude that $X_{\left\langle v^{+}\right\rangle,\left\langle v^{-}\right\rangle}=L_{X}$. Then $Q_{X} \cap L=Q_{X} \cap G_{\left\langle v^{+}\right\rangle,\left\langle v^{-}\right\rangle}=Q_{X} \cap L_{X}=1$.

Thus $Q_{X} \cap L=1$. Since $\operatorname{dim} Q_{X}=\operatorname{dim} Q=\operatorname{dim}(Y / L)$ by 2.1 (iii), it follows that $Q_{X}$ has a dense open orbit on $Y / L$. Now $Y / L$ is an affine irreducible variety as $L$ is reductive (see [Ha, Ri]). By [Bo, 4.10], every orbit of a unipotent group on an affine variety is closed. We deduce that $Q_{X}$ is transitive on $Y / L$ - that is, $Y=Q_{X} L$. Therefore $G=X Y=X Q_{X} L=X L$.

Proposition 2.3. Assume that $G$ is of exceptional type, and that $X$ is a reductive maximal closed connected subgroup of $G$.

(i) If $X$ is of maximal rank and $\operatorname{dim} X \geq \frac{1}{2} \operatorname{dim} G$, then $(G, X)$ is one of the following: $\left(E_{8}, A_{1} E_{7}\right),\left(E_{7}, A_{1} D_{6}\right),\left(F_{4}, B_{4}\right),\left(F_{4}, C_{4}\right)(p=2),\left(G_{2}, A_{2}\right),\left(G_{2}, \tilde{A}_{2}\right)$ $(p=3)$.

(ii) Suppose that $X$ is not of maximal rank, and that $\operatorname{dim} X$ is greater than $66,55,22,14$ or 3 , according as $G=E_{8}, E_{7}, E_{6}, F_{4}$ or $G_{2}$, respectively. Then $(G, X)$ is $\left(E_{6}, F_{4}\right),\left(E_{6}, C_{4}\right)(p \neq 2)$ or $\left(F_{4}, A_{1} G_{2}\right)(p \neq 2)$.

Proof. (i) Since $X$ is of maximal rank, it is generated by root groups corresponding to a subsystem $\Delta$ of the root system of $G$. As $X$ is maximal connected, it has no central torus (otherwise it lies in a Levi subgroup). It follows that, apart from the cases where $(G, p)=\left(F_{4}, 2\right)$ or $\left(G_{2}, 3\right), \Delta$ is obtained by deleting a node in the extended Dynkin diagram of $G$. Now a check of dimensions gives the conclusion. In the exceptional cases, the dual of such a subsystem $\Delta$ also yields a group $X$, giving the extra cases $\left(F_{4}, C_{4}\right)(p=2)$ and $\left(G_{2}, \tilde{A}_{2}\right)(p=3)$.

(ii) We use [Se2, Theorem 1], which determines the maximal connected subgroups $X$ of $G$ (assuming certain mild restrictions on the characteristic $p$ when $X$ is of small rank). By the lower bounds on $\operatorname{dim} X$, none of the characteristic restrictions comes into play, and the result is immediate from [Se2, Theorem 1].

The rest of this section contains various results on representations of simple algebraic groups with small dimensions, and corresponding subgroups of classical groups. We use the notation

$$
G=C l(V)
$$


to indicate that $G$ is a classical algebraic group with natural module $V$ (where if $(G, p)=\left(B_{n}, 2\right)$, we take $V$ to be the natural $2 n$-dimensional symplectic module).

The next proposition is a general result on maximal subgroups of classical groups, taken from $[\mathrm{Se} 1]$.

Proposition 2.4 ([Se1, Theorem 3]). Let $G=C l(V)$, and suppose that $X$ is a maximal closed connected subgroup of $G$. Then one of the following holds:

(i) $X=P_{k}$ or $N_{k}$ for some $k$ (notation as in the Introduction);

(ii) $V=U \otimes W$ and $X=C l(U) \otimes C l(W)$; $2)$;

(iii) $(X, G)=(S p(V), S L(V)),(S O(V), S L(V))(p \neq 2)$ or $(S O(V), S p(V))(p=$

(iv) $X$ is simple, and $V \downarrow X$ is irreducible and tensor indecomposable.

Proposition 2.5. Let $X$ be a simple algebraic group over the algebraically closed field $K$ of characteristic $p$, and suppose that $V=V_{X}(\lambda)$ is a rational irreducible $K X$-module such that $\operatorname{dim} V \leq \operatorname{dim} X$ and $X \neq C l(V)$. Then, up to duals and field twists, either $V$ is a composition factor of the adjoint module for $X$, or $X, \lambda$ are as follows:

\begin{tabular}{ll}
\hline$X$ & $\lambda$ \\
\hline$A_{n}$ & $\lambda_{2}, 2 \lambda_{1}, \lambda_{3}(n=5,6,7)$ \\
$C_{n}$ & $\lambda_{2}, \lambda_{3}(n=3), \lambda_{n}(3 \leq n \leq 6, p=2)$ \\
$B_{n}, D_{n}$ & $\lambda_{2}, \lambda_{n}(n \leq 7), \lambda_{n-1}\left(X=D_{n}, n \leq 7\right)$ \\
$G_{2}$ & $\lambda_{1}$ \\
$F_{4}$ & $\lambda_{4}$ \\
$E_{6}$ & $\lambda_{1}$ \\
$E_{7}$ & $\lambda_{7}$ \\
\hline
\end{tabular}

Proof. This is immediate from [Li, Section 2].

The next result determines the type of form (symplectic or quadratic) fixed by a simple algebraic group $X$ on a self-dual module in many cases. In the statement we use the usual parametrization $h_{\alpha}(t)$ for elements of a Cartan subgroup of $X$, where $t \in K^{*}$ and $\alpha$ is a root in the root system of $X$.

Proposition 2.6. Let $X$ be as in 2.5, and let $V_{X}(\lambda)$ be a rational irreducible selfdual $K X$-module.

(i) Suppose $p \neq 2$, and define $z=\prod h_{\alpha}(-1)$, the product being over all positive roots $\alpha$ of $X$. Then $X$ preserves a quadratic form on $V_{X}(\lambda)$ if and only if $\lambda(z)=1$.

(ii) Suppose $p=2$ and the Weyl module $W_{X}(\lambda)$ is irreducible (i.e. $V_{X}(\lambda)=$ $\left.W_{X}(\lambda)\right)$. Then either $(X, \lambda)=\left(C_{n}, \lambda_{1}\right)$, or $X$ preserves a quadratic form on $V_{X}(\lambda)$.

Proof. Part (i) is [St1, Lemma 79]. Part (ii) is proved in [KST]; as this is unpublished, we sketch the argument. Let $V=V_{X}(\lambda)$. The action of $X$ on $V$ gives a morphism $X \rightarrow S p_{2 n}$, where $\operatorname{dim} V=2 n$. Following this by a morphism $S p_{2 n} \rightarrow S O_{2 n+1}$ gives a morphism $X \rightarrow S O_{2 n+1}$. Let $W$ be the corresponding $(2 n+1)$-dimensional $X$-module. Then $W$ is an extension of a Frobenius twist $V^{\sigma_{2}}$ by the trivial $X$-module. If this extension is indecomposable, then by [LS2, 1.3], either $(X, \lambda)=\left(C_{n}, \lambda_{1}\right)$, or there is an indecomposable extension of $V$ by the trivial $X$-module, which contradicts the hypothesis that $V_{X}(\lambda)=W_{X}(\lambda)$. Therefore, assuming that $(X, \lambda) \neq\left(C_{n}, \lambda_{1}\right)$, we see that $W$ must be decomposable as $V^{\sigma_{2}} \oplus\langle v\rangle$. Therefore $X$ preserves a non-degenerate quadratic form on $V^{\sigma_{2}}$, hence on $V$. 
Proposition 2.7. Let $X$ be as in 2.5, and suppose $V=V_{X}(\lambda)$ is such that $X \neq$ $C l(V)$ and

$$
\operatorname{dim} V \leq \frac{1}{2}(\operatorname{dim} X-\operatorname{rank}(X)+4) .
$$

Then, up to duals and twists, $X, \lambda$ are as in the following table; we also give $C l(V)$, the smallest classical group on $V$ containing $X$ :

\begin{tabular}{lll}
\hline$X$ & $\lambda$ & $C l(V)$ \\
\hline$A_{n}(n \geq 4)$ & $\lambda_{2}$ & $S L_{n(n+1) / 2}$ \\
$B_{3}\left(\right.$ or $\left.C_{3}, p=2\right)$ & $\lambda_{3}$ & $S O_{8}$ \\
$B_{4}\left(\right.$ or $\left.C_{4}, p=2\right)$ & $\lambda_{4}$ & $S O_{16}$ \\
$D_{5}$ & $\lambda_{5}$ & $S L_{16}$ \\
$D_{6}$ & $\lambda_{5}$ or $\lambda_{6}$ & $S p_{32}(p \neq 2), S O_{32}(p=2)$ \\
$G_{2}$ & $\lambda_{1}$ & $S O_{7}(p \neq 2), S p_{6}(p=2)$ \\
$F_{4}$ & $\lambda_{4}$ & $S O_{26}(p \neq 3), S O_{25}(p=3)$ \\
$E_{6}$ & $\lambda_{1}$ & $S L_{27}$ \\
$E_{7}$ & $\lambda_{7}$ & $S p_{56}(p \neq 2), S O_{56}(p=2)$ \\
\hline
\end{tabular}

Proof. The possibilities for $\lambda$ are immediate from 2.5. Except for $(X, p)=\left(G_{2}, 2\right)$, the group $C l(V)$ is determined by 2.6. In the exceptional case it is clear that $X$ lies in $\mathrm{Sp}_{6}$ but not in $\mathrm{SO}_{6}$.

Proposition 2.8. Let $X$ be as in 2.5 and let $V=V_{X}(\lambda)$ with $\operatorname{dim} V \leq \operatorname{dim} X$. Assume that $p=2$ and that $X<S p(V)$ but $X \not \leq S O(V)$. Then either $V$ is a composition factor of the adjoint module for $X$, or $(X, \lambda)=\left(G_{2}, \lambda_{1}\right)$.

Proof. This follows from 2.5 and 2.6.

Our final result is also a straightforward consequence of 2.5.

Proposition 2.9. Let $X$ be as in 2.5, and assume that $V=V_{X}(\lambda)$ is self-dual and that $X \neq C l(V)$. Let $P=Q L$ be the stabilizer of a 1-space spanned by a maximal vector in $V$, where $Q$ is the unipotent radical and $L$ a Levi subgroup of the parabolic P. Suppose that

$$
\operatorname{dim} V \leq \max \left(\operatorname{dim} X-\operatorname{dim} L^{\prime}+1, \frac{1}{2} \operatorname{dim} X+2\right) .
$$

Then, up to duals and twists, $(X, \lambda)$ is one of the following: $\left(A_{2}, \lambda_{1}+\lambda_{2}\right),\left(A_{5}, \lambda_{3}\right)$, $\left(C_{3}, \lambda_{2}\right),\left(C_{3}, \lambda_{3}\right)(p \neq 2),\left(B_{n}, \lambda_{n}\right)(n \leq 5),\left(C_{n}, \lambda_{n}\right)(n \leq 5, p=2),\left(D_{6}, \lambda_{5}\right.$ or $\left.\lambda_{6}\right)$, $\left(G_{2}, \lambda_{1}\right),\left(F_{4}, \lambda_{4}\right),\left(E_{6}, \lambda_{1}\right),\left(E_{7}, \lambda_{7}\right)$.

\section{Exceptional groups: proof of Theorem A}

Let $G$ be a simple algebraic group of exceptional type in characteristic $p$, and assume that $G=X Y$ with $X, Y$ proper closed subgroups of $G$. By 1.1 we have $G=X^{0} Y^{0}$. Choose maximal connected subgroups $X_{1}, Y_{1}$ of $G$ containing $X^{0}, Y^{0}$ respectively. We aim to show that $\left(G, p, X_{1}, Y_{1}\right)$ is $\left(F_{4}, 2, B_{4}, C_{4}\right)$ or $\left(G_{2}, 3, A_{2}, \tilde{A}_{2}\right)$. Theorem A will follow quickly from this.

Lemma 3.1. Both $X_{1}$ and $Y_{1}$ are reductive. 
Proof. Suppose otherwise. Then $X_{1}$ or $Y_{1}$ is parabolic, say $Y_{1}$. Write $Y_{1}=Q L$, where $Q$ is the unipotent radical and $L$ a Levi subgroup. By 2.1(i,v), $X_{1}$ is reductive and is not of maximal rank in $G$. Also $\operatorname{dim} X_{1} \geq 2 \operatorname{dim}\left(G / Y_{1}\right)+\operatorname{rank}\left(X_{1}\right)$ by 2.1(iv). Inspection of $\operatorname{dim}\left(G / Y_{1}\right)$ for parabolics $Y_{1}$ yields

$$
\operatorname{dim}\left(G / Y_{1}\right) \geq 57,27,16,15,6 \text { for } G=E_{8}, E_{7}, E_{6}, F_{4}, G_{2} \text { (respectively). }
$$

Hence $\operatorname{dim} X_{1} \geq 114,54+\operatorname{rank}\left(X_{1}\right), 32,30,12$ in the respective cases. Now 2.3(ii) forces $G=E_{6}$ and $X_{1}=F_{4}$ or $C_{4}(p \neq 2)$.

By 2.1(ii,iii), $X_{1} \cap Y_{1}$ is a parabolic subgroup $Q_{X_{1}} L_{X_{1}}$ of $X_{1}$, with unipotent radical $Q_{X_{1}}$, Levi subgroup $L_{X_{1}}$, and $\operatorname{dim} Q_{X_{1}}=\operatorname{dim} Q=\operatorname{dim}\left(G / Y_{1}\right)$. Hence $Y_{1}$ is not $P_{3}, P_{4}$ or $P_{5}$ (as for these, $\operatorname{dim}\left(G / Y_{1}\right) \geq 25$, which is greater than the dimension of a maximal unipotent subgroup of $X_{1}=F_{4}$ or $C_{4}$ ). So $Y_{1}$ is $P_{1}, P_{2}$ or $P_{6}$; and if $Y_{1}=P_{2}$ then $\operatorname{dim}\left(G / Y_{1}\right)=21$ forces $X_{1}=F_{4}$.

Suppose $Y_{1}=P_{2}$. Then by 2.2 ,

$$
G=E_{6}=X_{1} L=F_{4} A_{5} T_{1} .
$$

Now $L=A_{5} T_{1}$ normalizes a fundamental subgroup $A_{1}$ of $G$. As $X_{1}=F_{4}$ contains a conjugate of this $A_{1}$, this contradicts 1.1(iii).

Thus $Y_{1}=P_{1}$ or $P_{6}$, and $\operatorname{dim}\left(G / Y_{1}\right)=16$. One checks that 16 is not the dimension of the unipotent radical of any parabolic subgroup of $F_{4}$. Hence $X_{1}=$ $C_{4}(p \neq 2)$ and $X_{1} \cap Y_{1}$ is a Borel subgroup of $X_{1}$. However both $X_{1}$ and $Y_{1}$ then contain fundamental subgroups $S L_{2}$ of $G$; so replacing $X, Y$ by conjugates, we may take $S L_{2} \leq X_{1} \cap Y_{1}$, which contradicts the previous sentence.

Lemma 3.2. We have $\left(G, p, X_{1}, Y_{1}\right)=\left(F_{4}, 2, B_{4}, C_{4}\right)$ or $\left(G_{2}, 3, A_{2}, \tilde{A}_{2}\right)$.

Proof. Since $G=X_{1} Y_{1}$, we may assume that $\operatorname{dim} X_{1} \geq \frac{1}{2} \operatorname{dim} G$.

Suppose first that $X_{1}$ is not of maximal rank in $G$. Then by 2.3(ii), $G=E_{6}$ and $X_{1}=F_{4}$. Hence $\operatorname{dim} Y_{1} \geq 26$, so again by 2.3 , either $Y_{1}$ is of maximal rank or $Y_{1}=F_{4}$ or $C_{4}(p \neq 2)$. If $Y_{1}$ is of maximal rank, then $\operatorname{dim} Y_{1} \geq 26$ forces $Y_{1}=A_{1} A_{5}$. But then $Y_{1}$ normalizes a fundamental $A_{1}$, a conjugate of which lies in $X_{1}$, contrary to 1.1(iii). If $Y_{1}=F_{4}$, then $X_{1}$ and $Y_{1}$ are $G$-conjugate, which is impossible. Thus $Y_{1}=C_{4}(p \neq 2)$. Then $\operatorname{dim}\left(X_{1} \cap Y_{1}\right)=10$. By [CLSS, 2.7], we can choose $X_{1}, Y_{1}$ so that $X_{1}=C_{G}(\tau), Y_{1}=C_{G}(\tau h)$, where $\tau$ is a graph automorphism of $G$ and $h$ is an involution of $G$ commuting with $\tau$. Then $X_{1} \cap Y_{1}=C_{G}(\tau, h)=C_{X_{1}}(h)=A_{1} C_{3}$, which has dimension more than 10 , a contradiction.

This establishes that $X_{1}$ must have maximal rank. Then $X_{1}$ is given by 2.3(i): $X_{1}$ is $A_{1} E_{7}, A_{1} D_{6}, B_{4}$ or $C_{4}(p=2), A_{2}$ or $\tilde{A}_{2}(p=3)$, according as $G=E_{8}, E_{7}, F_{4}, G_{2}$, respectively. We deduce that $\operatorname{dim} Y_{1}$ is at least 112, 64, 16, 6 in the respective cases. If $G=E_{7}$ or $E_{8}$ then 2.3(ii) forces $Y_{1}$ to be of maximal rank also. But then $X_{1}$ normalizes a fundamental $A_{1}$, a conjugate of which lies in $Y_{1}$, a contradiction.

Now let $G=F_{4}$. By 2.3(ii), either $Y_{1}$ is of maximal rank or $Y_{1}=A_{1} G_{2}(p \neq$ 2 ). In the latter case, $Y_{1}$ normalizes the factor $G_{2}$, while $X_{1}=B_{4}$ contains a conjugate of this $G_{2}$, contrary to 1.1(iii). Hence $Y_{1}$ is of maximal rank, and so $Y_{1}$ is $B_{4}, C_{4}(p=2), A_{1} C_{3}, \tilde{A}_{1} B_{3}, A_{2} \tilde{A}_{2}, A_{3} \tilde{A}_{1}$ or $\tilde{A}_{3} A_{1}$. The last five cases are impossible by 1.1(iii), as $X_{1}$ contains conjugates of the factors $A_{1}$ or $A_{2}$ of these maximal rank subgroups. Therefore, as $Y_{1}$ is not conjugate to $X_{1}$, we conclude that $p=2$ and $\left\{X_{1}, Y_{1}\right\}=\left\{B_{4}, C_{4}\right\}$, as in the conclusion. 
Finally, consider $G=G_{2}$. Here $Y_{1}$ is of maximal rank by 2.3(ii), so $Y_{1}$ is $A_{2}, \tilde{A}_{2}(p=3)$ or $A_{1} \tilde{A}_{1}$. The latter is impossible by 1.1(iii) as usual, so $p=3$ and $\left\{X_{1}, Y_{1}\right\}=\left\{A_{2}, \tilde{A}_{2}\right\}$, as required.

To complete the proof of Theorem A, it remains to determine the possibilities for the (not necessarily maximal) connected subgroups $X^{0}, Y^{0}$ lying in $X_{1}, Y_{1}$, such that $G=X^{0} Y^{0}$. Observe that $X_{1}=X^{0}\left(X_{1} \cap Y_{1}\right)$.

If $G=G_{2}$ and $\left(X_{1}, Y_{1}\right)=\left(A_{2}, \tilde{A}_{2}\right)$, then $\operatorname{dim}\left(X_{1} \cap Y_{1}\right)=2$, so $X^{0}$ (respectively $Y^{0}$ ) can have codimension at most 2 in $X_{1}$ (respectively $Y_{1}$ ). But the only proper connected subgroup of $A_{2}$ of codimension 2 or less is a parabolic, and $G_{2}$ has no parabolic factorizations by 3.1. Hence $X^{0}=X_{1}, Y^{0}=Y_{1}$. This does give a factorization of $G_{2}$, by 1.9 .

Finally, consider $G=F_{4},\left(X_{1}, Y_{1}\right)=\left(B_{4}, C_{4}\right)$ (with $p=2$ ). Suppose that $X^{0}<X_{1}$. Since $G=X^{0} Y_{1}$, we have $\operatorname{dim} X^{0} \geq 16$; also $X^{0}$ lies in no parabolic subgroup of $G$, by 3.1. Using 2.4 and 2.5, we see that this forces $X^{0}=D_{4}$ or $D_{3} B_{1}$. In the latter case $Y_{1}$ contains a conjugate of the subgroup $B_{1}$ (note that $B_{1}=\tilde{A}_{1}$ ), contrary to 1.1(iii). Hence $X^{0}=D_{4}$.

We have established that $X^{0}=B_{4}$ or $D_{4}$; similarly, $Y^{0}=C_{4}$ or $\tilde{D}_{4}$. All these possibilities give factorizations of $F_{4}$, by 1.9 .

This completes the proof of Theorem A.

\section{Classical groups: parabolic factorizations}

In this section we determine the maximal parabolic factorizations of classical algebraic groups, showing that they are as in (1) of Theorem B.

Let $G$ be a simple algebraic group of classical type, with natural module $V$ over an algebraically closed field $K$ of characteristic $p$. If $(G, p)=\left(B_{n}, 2\right)$, we take $V$ to be the $2 n$-dimensional symplectic module; and if $G=D_{n}$, we assume that $n \geq 4$. Suppose that $G=X Y$, where $X, Y$ are maximal closed connected subgroups of $G$ and $Y$ is parabolic. Thus $Y=P_{i}$ for some $i$, the parabolic obtained by deleting the $i$ th node from the Dynkin diagram of $G$. Write $Y=Q L$, where $Q$ is the unipotent radical and $L$ a Levi subgroup of $Y$. We aim to show that $G, X, Y$ are as in (1) of Theorem B. Since those factorizations exist by 1.2 , this will establish Theorem B for parabolic factorizations.

By 2.1, $X$ is reductive and $X \cap Y=P_{X}=Q_{X} L_{X}$, a parabolic subgroup of $X$ with unipotent radical $Q_{X}$ and Levi subgroup $L_{X}$. Moreover, $\operatorname{dim} Q_{X}=\operatorname{dim} Q=$ $\operatorname{dim}(G / Y)$.

Our first lemma is immediate from inspection of parabolic subgroups of $G$.

Lemma 4.1. Define a number $c(G)$ as follows: $c(G)=1$ if $G \neq D_{n}, c(G)=2$ if $G=D_{n}$. Then $\operatorname{dim} Q \geq \operatorname{dim} V-c(G)$.

In the rest of the section we consider separately the possibilities for $X$ which are given by 2.4 .

Lemma 4.2. If $X$ is simple and irreducible on $V$, and $X \neq S p(V), S O(V)$, then $(G, X, Y)=\left(S O_{8}, B_{3}, P_{i}\right)(i=1,3,4),\left(S O_{7}, G_{2}, P_{1}\right)(p \neq 2)$ or $\left(S p_{6}, G_{2}, P_{1}\right)(p=$ 2) (as in (1) of Theorem $B$ ). 
Proof. Suppose $X$ is simple and $V$ is the irreducible $K X$-module $V_{X}(\lambda)$. By 2.1(iv), we have $\operatorname{dim} X \geq 2 \operatorname{dim} Q+\operatorname{rank}(X)$. Hence by 4.1 ,

$$
\operatorname{dim} V \leq \operatorname{dim} Q+c(G) \leq \frac{1}{2}(\operatorname{dim} X-\operatorname{rank}(X)+4) .
$$

Consequently the possibilities for $(X, \lambda)$ are given by 2.7 ; and since $X$ is maximal, $G$ is the group $C l(V)$ given in 2.7.

Assume first that $Y=P_{1}\left(\right.$ or $P_{n-1}$ for $\left.G=S L_{n}\right)$. Then $\operatorname{dim} Q=\operatorname{dim} V-c(G)$, hence also $\operatorname{dim} Q_{X}=\operatorname{dim} V-c(G)$. The parabolic $X \cap Y=P_{X}$ is the stabilizer in $X$ of a 1-space spanned by a maximal vector of $V$ (or of $V^{*}$ if $Y=P_{n-1}$ ), hence is given by deleting from the Dynkin diagram of $X$ those nodes corresponding to nonzero coefficients in $\lambda$. We conclude from the list in 2.7 that $\operatorname{dim} Q_{X}$ is as follows:

$$
\begin{array}{cccccccccc}
X= & A_{n} & B_{3} & B_{4} & D_{5} & D_{6} & G_{2} & F_{4} & E_{6} & E_{7} \\
\lambda= & \lambda_{2} & \lambda_{3} & \lambda_{4} & \lambda_{5} & \lambda_{5}, \lambda_{6} & \lambda_{1} & \lambda_{4} & \lambda_{1} & \lambda_{7} \\
\operatorname{dim} Q_{X}= & 2 n-2 & 6 & 10 & 10 & 15 & 5 & 15 & 16 & 27
\end{array}
$$

The fact that $\operatorname{dim} Q_{X}=\operatorname{dim} V-c(G)$ now forces $(X, G)$ to be $\left(B_{3}, S O_{8}\right),\left(G_{2}, S O_{7}\right)$ $(p \neq 2)$ or $\left(G_{2}, S p_{6}\right)(p=2)$.

Now suppose that $Y=P_{i}$ with $i \geq 2$ (and $i \leq n-2$ if $G=S L_{n}$ ). Inspection of the list in 2.7 shows that $\operatorname{dim} Q$ is greater than the dimension of a maximal unipotent subgroup of $X$, except in the following cases:

$$
\begin{gathered}
(G, X, Y)=\left(S O_{7}, G_{2}, P_{3}\right) \quad(p \neq 2),\left(S p_{6}, G_{2}, P_{3}\right) \quad(p=2), \\
\left(S O_{8}, B_{3}, P_{i}\right) \quad(i=2,3,4) .
\end{gathered}
$$

Since $\operatorname{dim} Q=\operatorname{dim} Q_{X}$, one of these cases must occur. It remains to rule out the cases $(G, X, Y)=\left(S O_{7}, G_{2}, P_{3}\right),\left(S p_{6}, G_{2}, P_{3}\right)$ and $\left(S O_{8}, B_{3}, P_{2}\right)$. In the first two cases, 2.2 implies that $S O_{7}=G_{2} A_{2} T_{1}$ (where $A_{2} T_{1}$ is a Levi subgroup of $P_{3}$ ). But the subgroup $G_{2}$ contains a conjugate of this $A_{2}$, so this is impossible. And in the last case, application of triality yields $S O_{8}=N_{1} P_{2}$. This implies that $N_{1}$, the stabilizer of a nonsingular vector $v$, is transitive on the set of totally singular 2 -spaces, which is false (since a totally singular 2-space may or may not lie in $\left.v^{\perp}\right)$.

Lemma 4.3. If $X$ is $S p(V)$ or $S O(V)$, then $(G, X, Y)=\left(S L_{2 m}, S p_{2 m}, P_{1}\right.$ or $\left.P_{2 m-1}\right)$ (as in Theorem $B(1)$ ).

Proof. If $G=S L(V)$, then the fact that $G=X P_{i}$ implies that $X$ is transitive on $i$-spaces in $V$, and clearly the only possibility is that given in the conclusion. Otherwise, $G=S p(V), X=S O(V), p=2$ and $X$ is transitive on totally isotropic $i$-spaces in $V$. This is impossible, as some of these $i$-spaces are totally singular (with respect to the quadratic form on $V$ preserved by $X$ ), and some are not.

Lemma 4.4. Suppose that $X=N_{k}$, the (connected) stabilizer in $G$ of a nondegenerate $k$-subspace of $V$ (or, if $(G, p)=\left(D_{n}, 2\right)$, of a nonsingular 1-space). Then $(G, X, Y)=\left(S O_{2 m}, N_{1}, P_{m}\right.$ or $\left.P_{m-1}\right)$ (as in Theorem $\left.B(1)\right)$.

Proof. By 2.1(v), $X$ is not of maximal rank in $G$. Hence $G=D_{m}$ and $X=$ $B_{l} B_{m-l-1}$, where $k=2 l+1 \leq m$. Also, if $p=2$, then $k=1, l=0$. Let $X$ be the stabilizer of the $k$-subspace $W$ of $V$. Thus either $W$ is non-degenerate, or $p=2$ and $W$ is a nonsingular 1-space.

Recall that $Y=P_{i}$. Suppose that $i \leq m-2$. Then $X$ is transitive on totally singular $i$-spaces in $V$. However, if $k>1$, then there exist totally singular $i$-spaces 
$W_{1}, W_{2}$ such that $W_{1} \cap W, W_{2} \cap W$ have dimensions $l, l-1$, respectively; and if $k=1$ there exist totally singular $i$-spaces $W_{1}, W_{2}$ such that $W_{1} \subseteq W^{\perp}, W_{2} \nsubseteq W^{\perp}$. Hence $X$ cannot in fact be transitive, a contradiction.

Therefore $i=m$ or $m-1$, and $Y$ is the stabilizer of a totally singular $m$-space $U$. If $k>1$, then there are non-degenerate $k$-spaces $U_{1}, U_{2}$ such that $U_{1} \cap U, U_{2} \cap U$ have dimensions $l, l-1$, respectively; hence $Y$ is not transitive on non-degenerate $k$-spaces, a contradiction.

We conclude that $i=m$ or $m-1$ and $k=1$, as required.

By Lemmas 4.2, 4.3, 4.4 and Proposition 2.4, the only remaining possibility for the maximal connected subgroup $X$ is

$$
X=C l(U) \otimes C l(W), \text { where } V=U \otimes W .
$$

The various subgroups $X<G$ occurring are as follows:

$$
\begin{aligned}
& S L_{a} \otimes S L_{b}<S L_{a b}, \\
& S p_{a} \otimes S p_{b}<S O_{a b}, \\
& S p_{a} \otimes S O_{b}<S p_{a b}, \\
& S O_{a} \otimes S O_{b}<S O_{a b} .
\end{aligned}
$$

Note that for any factor $S O_{a}$ or $S O_{b}$ we have $a \geq 3$ or $b \geq 3$ accordingly, since $S_{2}$ is a reducible group.

For convenience in the proof of Theorem B, we handle in the next lemma all factorizations $G=X Y$, where $Y$ is either $P_{i}$ (as assumed at the beginning of this section) or $N_{i}$.

Lemma 4.5. Let $G=C l(V), X=C l(U) \otimes C l(W)$ with $V=U \otimes W$. Suppose that $G=X Y$ with $Y=P_{i}$ or $N_{i}$. Then $(G, X, Y)=\left(S O_{2 a}, S p_{a} \otimes S p_{2}, N_{1}\right)$ with a even. In particular, no parabolic factorizations occur in this case.

Proof. Let $a=\operatorname{dim} U, b=\operatorname{dim} W$, and for any $m \leq a, n \leq b$ let $U_{m}, W_{n}$ denote subspaces of $U, W$ of dimension $m, n$ respectively. Also, write $U(X)$ for a maximal connected unipotent subgroup of $X$.

The proof is somewhat long and tedious, and we divide it into a number of steps.

(1) $X$ is not $S L_{a} \otimes S L_{b}$.

For suppose that $X=S L_{a} \otimes S L_{b}, G=S L_{a b}$, and take $a \geq b$. Then $Y=P_{i}$, and $\operatorname{dim} Q=\operatorname{dim} Q_{X}=i(a b-i)$. We may assume that $i \leq \frac{1}{2} a b$ (since $G=X P_{i}$ implies $G=X P_{a b-i}$ by application of a graph automorphism).

If $i \geq a$, then $\operatorname{dim} Q_{X} \geq a(a b-a)$, and hence $a(a b-a) \leq \operatorname{dim} U(X)=$ $\frac{1}{2} a(a-1)+\frac{1}{2} b(b-1)<a^{2}$, which is false. Therefore $i<a$. Now define $i$-subspaces $A, B$ of $V$ as follows. If $i>1$,

$$
A=U_{i} \otimes w, \quad B=\left(U_{i-1} \otimes w\right) \oplus\left\langle u \otimes w^{\prime}\right\rangle,
$$

where $u \in U-U_{i-1}$ and $w, w^{\prime} \in W$ are linearly independent; and if $i=1$,

$$
A=\langle u \otimes w\rangle, \quad B=\left\langle u \otimes w+u^{\prime} \otimes w^{\prime}\right\rangle,
$$

where $u, u^{\prime} \in U$ and $w, w^{\prime} \in W$ are linearly independent. Then $A$ and $B$ lie in different $X$-orbits, and hence $G \neq X P_{i}$, a contradiction.

(2) (i) If $Y=P_{i}$ and $X=S p_{a} \otimes S p_{b}$ or $S O_{a} \otimes S O_{b}$ with $a \geq b$, then $i<\frac{1}{2}(a-1)$.

(ii) If $Y=P_{i}$ and $X=S p_{a} \otimes S O_{b}$, then $i<\frac{1}{2} a$ if $a \geq b$, and $i<\frac{1}{2}(b-1)$ if $a \leq b$. 
We prove (i). Here $G=S O_{a b}$ and $\operatorname{dim} Q_{X}=\operatorname{dim} Q=i\left(a b-\frac{3}{2} i-\frac{1}{2}\right)=d_{i}$, say. Note that $a \geq 3$, since otherwise $X=S p_{2} \otimes S p_{2}=S O_{4}=G$.

Suppose that $i \geq \frac{1}{2}(a-1)$. Now $d_{i}$ increases with $i$ until reaching a maximum at $i=x$ or $x+1$, where $x=\left[\frac{1}{3}\left(a b-\frac{1}{2}\right)\right]$, after which $d_{i}$ decreases. Hence either $\operatorname{dim} Q \geq d_{\left[\frac{1}{2}(a-1)\right]}$ or $\operatorname{dim} Q \geq d_{\left[\frac{1}{2} a b\right]}$. In the first case, we have

$$
\frac{1}{2}(a-1)(a b-a+1) \leq \operatorname{dim} Q_{X} \leq \operatorname{dim} U(X) \leq \frac{1}{4}\left(a^{2}+b^{2}\right) .
$$

This implies that $a=b=2$, a contradiction. We obtain a similar contradiction when $\operatorname{dim} Q \geq d_{\left[\frac{1}{2} a b\right]}$.

For part (ii), note that here $\operatorname{dim} Q=i\left(a b-\frac{3}{2} i+\frac{1}{2}\right)$ and apply the same argument.

(3) $Y=P_{i}$ does not occur.

For suppose $Y=P_{i}$. Then (2)(i) or (2)(ii) holds. Interchanging the factors in (ii) if necessary, we can assume that $a \geq b$, and then (2) gives $i<\frac{1}{2} a$, and also $i<\frac{1}{2}(a-1)$ if $a$ is odd. Now define totally singular $i$-subspaces $A, B$ of $V$ as in the proof of (1) above (taking $U_{i}$ and $\left\langle U_{i-1}, u\right\rangle$ to be totally singular in $U$, etc.). Then $A, B$ are in different $X$-orbits, so $G \neq X P_{i}$.

In view of (3), we have $Y=N_{i}$. We may assume that $i \leq \frac{1}{2} a b$. Interchanging the factors of $X$ if necessary, we may also take $a \geq b$.

(4) We have $i \leq 2 a$.

To see this, suppose first that $G=S O_{a b}$, so that $X=S p_{a} \otimes S p_{b}$ or $S O_{a} \otimes S O_{b}$. Then $\operatorname{dim}\left(G / N_{i}\right)=i(a b-i)$. If $i>2 a$, then $b \geq 5\left(\right.$ as $\left.i \leq \frac{1}{2} a b\right)$, so $\operatorname{dim} X \geq$ $\operatorname{dim}\left(G / N_{i}\right)>2 a(a b-2 a)$, which is false. Therefore $i \leq 2 a$ in this case. A similar argument proves this when $G=S p_{a b}$.

(5) We have $b=2$.

For suppose $b \geq 3$. Choose a non-degenerate 2-space $W_{2}$ in $W$. Then $U \otimes W_{2}$ is a non-degenerate $2 a$-space in $V$. By (4) we may choose a non-degenerate $i$-space $A \subseteq U \otimes W_{2}$ (or, if $p=2$ and $i=1$, a nonsingular 1-space $A \subseteq U \otimes W_{2}$ ), and take $Y=\left(G_{A}\right)^{0}$. However, when $b \geq 3$ it is easy to see that there are nonsingular vectors which do not lie in any such subspace $U \otimes W_{2}$. Hence $X$ cannot be transitive on $G / N_{i}$. This shows that $b=2$.

By (5), $X=S p_{a} \otimes S p_{2}$ or $S O_{a} \otimes S p_{2}(p \neq 2)$. To complete the proof of 4.5, it only remains to show that $i=1$, that is, $Y=N_{1}$ (note that $N_{1}$ does not exist when $\left.X=S O_{a} \otimes S p_{2}, G=S p_{2 a}\right)$.

Suppose then that $i \geq 2$, and choose non-negative integers $q, r$ such that

$$
i=4 q+r \text { and } r \in\{0,2,3,5\} .
$$

(6) Either $2 q \leq a-4$ or $i=4$.

To see this, observe first that $i \leq \frac{1}{2} \operatorname{dim} V=a$, so $2 q=\frac{1}{2}(i-r) \leq \frac{1}{2}(a-r)$. If $2 q>a-4$, then $a<8$, so $q=0$ or 1 , and hence either $i=2$ and $X=S O_{3} \otimes S p_{2}$, or $i=4$. If $i=2$ and $X=S O_{3} \otimes S p_{2}$, then there is a non-degenerate $i$-space of the form $\langle u\rangle \otimes W_{2}$; since not all non-degenerate $i$-spaces are of this form, $X$ is not transitive on $G / N_{i}$, a contradiction. Therefore $i=4$ if $2 q>a-4$.

We now complete the proof. Pick a non-degenerate $2 q$-space $U_{2 q}$ in $U$, and set $M=U_{2 q} \otimes W$, a non-degenerate $4 q$-space in $V$. If $r=0$, then $i=4 q$ and $M$ is a non-degenerate $i$-space; however not all non-degenerate $i$-spaces are of this form, so $G \neq X N_{i}$ here. Therefore $r \neq 0$, and in particular $i \neq 4$. Consequently $2 q \leq a-4$ by (6). 
We may choose non-degenerate subspaces $U_{2}, U_{4}$ of $U$ such that $U_{2} \subseteq U_{4} \subseteq U_{2 q}^{\perp}$. If $r=2$ or 3 , choose non-degenerate $r$-subspaces $M_{1}, M_{2}$ in $V$ such that $M_{1} \subseteq$ $U_{2} \otimes W, M_{2} \subseteq U_{4} \otimes W$, and $M_{2}$ lies in no subspace $U_{2}^{\prime} \otimes W$ with $U_{2}^{\prime}$ a nondegenerate 2 -space in $U$. Then $M \oplus M_{1}$ and $M \oplus M_{2}$ are non-degenerate $i$-spaces lying in different $X$-orbits, so $G \neq X N_{i}$.

This leaves the case where $r=5$. Here $i=4 q+5$, an odd number, so $G$ must be $S O_{2 a}$ with $p \neq 2$, and $X=S p_{a} \otimes S p_{2}$. There exist non-degenerate $i$-spaces lying in $\left(U_{2 q}+U_{4}\right) \otimes W$, but not all non-degenerate $i$-spaces lie in a subspace of this form (i.e. of the form $U_{2 q+4} \otimes W$ ). Hence again $G \neq X N_{i}$.

We have now established that $i=1$ and $X=S p_{a} \otimes S p_{2}$, as in the conclusion of Lemma 4.5.

Lemmas 4.2-4.5 establish Theorem B for parabolic factorizations.

\section{Classical Groups: NOn-PARABOlic FaCtorizations}

Continue to assume that $G$ is a classical algebraic group with natural module $V$ over the algebraically closed field $K$ of characteristic $p$. Suppose that $G=$ $X Y$, where $X$ and $Y$ are maximal closed connected reductive subgroups of $G$. To complete the proof of Theorem B, we must show that $G, X, Y$ are as in (2), (3) or (4) of Theorem B (all these factorizations exist, by $\S 1$ ).

Clearly either $X$ or $Y$ has dimension at least $\frac{1}{2} \operatorname{dim} G$, say

$$
\operatorname{dim} X \geq \frac{1}{2} \operatorname{dim} G
$$

Lemma 5.1. The possibilities for $X, G$ are as follows:

\begin{tabular}{cc}
\hline$X$ & $G$ \\
\hline$N_{k}$ & $S p(V)$ or $S O(V)$ \\
$S p(V)$ & $S L(V)$ \\
$S O(V)$ & $S p(V)(p=2)$ \\
$B_{3}$ & $S O_{8}$ \\
$G_{2}$ & $S p_{6}(p=2), S O_{7}(p \neq 2)$ \\
\hline
\end{tabular}

Proof. The maximal subgroup $X$ of $G$ satisfies one of the conclusions of 2.4. If 2.4(i),(ii) or (iii) holds, then the fact that $\operatorname{dim} X \geq \frac{1}{2} \operatorname{dim} G$ forces $X$ to be $N_{k}, S p(V)$ or $S O(V)(p=2)$. In case (iv) of 2.4, $X$ is simple and irreducible on $V$; then $\operatorname{dim} X \geq \frac{1}{2} \operatorname{dim} G$ forces $\operatorname{dim} V \leq \operatorname{dim} X$, whence $X$ is given by 2.5. A quick check shows that $\operatorname{dim} X \geq \frac{1}{2} \operatorname{dim} G$ only in the cases given in the conclusion.

Remark. When $X=C_{3}$ and $V=V_{X}\left(\lambda_{3}\right)$ with $p=2$, the image of $X$ in $S O(V)$ is $B_{3}$ rather than $C_{3}$. Hence the pair $(X, G)=\left(C_{3}, S O_{8}\right)$ does not appear in the conclusion of 5.1 .

The remaining lemmas deal with the possibilities for $X$ given by 5.1 .

Lemma 5.2. If $X=N_{k}$, then $G, X, Y$ are as in Theorem $B(2,3,4)$.

Proof. Here $G=S p(V)$ or $S O(V)$, and we may take $k \leq \frac{1}{2} \operatorname{dim} V$.

We exclude until later the case where $k=1$ and $Y$ is simple and irreducible on $V$ with $Y \neq C l(V)$. Thus we assume now that either $k \geq 2$ or $Y$ satisfies (i), (ii) or (iii) of 2.4. When $k \geq 2$ and $Y$ is simple and irreducible, we have 
$\operatorname{dim} Y \geq \operatorname{dim}\left(G / N_{2}\right)=2 \operatorname{dim} V-4$, so $\operatorname{dim} V \leq \frac{1}{2} \operatorname{dim} Y+2$. By 2.4, we conclude that one of the following holds:

(a) $Y=N_{l}$ for some $l$,

(b) $Y=C l\left(V_{1}\right) \otimes C l\left(V_{2}\right)$, where $V=V_{1} \otimes V_{2}$,

(c) $Y=S O(V)$ with $G=S p(V), p=2$,

(d) $Y$ is simple and irreducible on $V$, with $k \geq 2$ and $\operatorname{dim} V \leq \frac{1}{2} \operatorname{dim} Y+2$.

In case (a), take $k \leq l$ and let $Y$ stabilize the $l$-space $W$. There are nonsingular $k$-spaces lying in $W$, and nonsingular $k$-spaces not lying in $W$, so $Y$ cannot be transitive on nonsingular $k$-spaces. Thus $G \neq N_{k} Y$, a contradiction.

In case (b), Lemma 4.5 implies that $k=1, Y=S p_{a} \otimes S p_{2}$ and $G=S O_{2 a}$, as in Theorem $\mathrm{B}(2)$. And in case (c) we have the factorization $S p(V)=S O(V) N_{2 m}$ (where $2 m=k$ ), as in Theorem $\mathrm{B}(4)$.

Assume now that (d) holds. Let $V \downarrow Y=V_{Y}(\lambda)$. Since $\operatorname{dim} V \leq \frac{1}{2} \operatorname{dim} Y+2$, the possibilities for $(Y, \lambda)$ are among those listed in the conclusion of 2.9 , from which we deduce that $Y, \lambda, G$ are in the following list:

\begin{tabular}{ccc}
\hline$Y$ & $\lambda$ & $G$ \\
\hline$B_{3}$ & $\lambda_{3}$ & $S O_{8}$ \\
$B_{4}$ & $\lambda_{4}$ & $S O_{16}$ \\
$D_{6}$ & $\lambda_{5}$ or $\lambda_{6}$ & $S p_{32}(p \neq 2), S O_{32}(p=2)$ \\
$G_{2}$ & $\lambda_{1}$ & $S O_{7}(p \neq 2), S p_{6}(p=2)$ \\
$F_{4}$ & $\lambda_{4}$ & $S O_{26}(p \neq 3), S O_{25}(p=3)$ \\
$E_{7}$ & $\lambda_{7}$ & $S p_{56}(p \neq 2), S O_{56}(p=2)$ \\
\hline
\end{tabular}

We have $k \geq 2$. Suppose that $k=2$. Then $G=S p(V)$ (as $N_{2}$ is non-maximal in $S O(V))$, so $Y=D_{6}, G_{2}$ or $E_{7}$. For $Y=G_{2}$ we have the factorization $S p_{6}=G_{2} N_{2}$ in Theorem $\mathrm{B}(4)$. For $Y=D_{6}$ or $E_{7}$, let $v^{+}$be a maximal vector in $V$. The stabilizer of $v^{+}$in $Y$ is a parabolic subgroup $Q L$, where $Q$ is the unipotent radical and $L$ a Levi subgroup (and $L^{\prime}=A_{5}$ or $E_{6}$, respectively). Relative to a maximal torus of $L$, let $\bar{w}_{0}$ be an element in the coset of the longest element of $W(Y)$, the Weyl group of $Y$, and let $v^{-}=v^{+} \bar{w}_{0}$. Then $\left\langle v^{+}, v^{-}\right\rangle$is a non-degenerate 2-space in $V$ which is stabilized by $L$. We conclude that $X \cap Y=N_{2} \cap Y$ contains $L^{\prime}=A_{5}$ or $E_{6}$. Thus $\operatorname{dim} X \cap Y>\operatorname{dim} X+\operatorname{dim} Y-\operatorname{dim} G$, and so $G \neq X Y$.

Now assume that $k \geq 3$. We know that $\operatorname{dim} Y \geq \operatorname{dim}\left(G / N_{k}\right)$. From the above table, the only possibilities for $(Y, \lambda)$ are $\left(B_{3}, \lambda_{3}\right)$ and $\left(G_{2}, \lambda_{1}\right)$. The factorization $S_{8}=B_{3} N_{3}$ is in Theorem $\mathrm{B}(2)$. Thus it remains to exclude the possibilities $(G, X, Y)=\left(S O_{8}, N_{4}, B_{3}\right)$ and $\left(S_{7}, N_{3}, G_{2}\right)$. In the first case, application of triality to a factorization $S O_{8}=B_{3} N_{4}$ would yield $S O_{8}=N_{1} N_{4}$, which is not true. Now suppose $S O_{7}=G_{2} N_{3}$. A maximal rank subgroup $A_{1} A_{1}$ of $G_{2}$ fixes a non-degenerate 3 -space, so lies in $X \cap Y$; but then $\operatorname{dim} X \cap Y=6>\operatorname{dim} X+$ $\operatorname{dim} Y-\operatorname{dim} G$, a contradiction.

We now consider the case where $k=1$ and $Y$ is simple and irreducible on $V$ with $Y \neq C l(V)$, excluded from consideration earlier. Here $G=S O(V)$ and $\operatorname{dim}(G / X)=\operatorname{dim}\left(G / N_{1}\right)=\operatorname{dim} V-1$. As above, let $v^{+}$be a maximal vector in $V$ with $Y_{v^{+}}=Q L$, a parabolic subgroup of $Y$, and let $v^{-}=v^{+} \bar{w}_{0}$. Then $\left\langle v^{+}, v^{-}\right\rangle$is a non-degenerate 2 -space in $V$, fixed pointwise by $L^{\prime}$. Taking $X$ to be the stabilizer in $G$ of a nonsingular 1-space in $\left\langle v^{+}, v^{-}\right\rangle$, we then have $L^{\prime} \leq X \cap Y$. Thus

$$
\operatorname{dim} Y=\operatorname{dim}(G / X)+\operatorname{dim} X \cap Y \geq \operatorname{dim} V-1+\operatorname{dim} L^{\prime} .
$$


Consequently $\operatorname{dim} V \leq \operatorname{dim} Y-\operatorname{dim} L^{\prime}+1$, and so the possibilities for $Y, \lambda$ (where $\left.V \downarrow Y=V_{Y}(\lambda)\right)$ are given by 2.9 ; as $G=S O(V)$, combining this with 2.6 shows that the possibilities are:

\begin{tabular}{lcl}
\hline$Y$ & $\lambda$ & $G$ \\
\hline$A_{2}$ & $\lambda_{1}+\lambda_{2}$ & $S O_{8-\delta_{p, 3}}$ \\
$A_{5}$ & $\lambda_{3}$ & $S O_{20}(p=2)$ \\
$C_{3}$ & $\lambda_{2}$ & $S O_{14-\delta_{p, 3}}$ \\
$B_{n}$ & $\lambda_{n}$ & $S O_{2^{n}}(n=3,4,5)$ \\
$D_{6}$ & $\lambda_{5}, \lambda_{6}$ & $S O_{32}(p=2)$ \\
$G_{2}$ & $\lambda_{1}$ & $S O_{7}(p \neq 2)$ \\
$F_{4}$ & $\lambda_{4}$ & $S O_{26-\delta_{p, 3}}$ \\
$E_{7}$ & $\lambda_{7}$ & $S O_{56}(p=2)$ \\
\hline
\end{tabular}

All these give factorizations $G=Y N_{1}$ in Theorem $\mathrm{B}$, apart from the cases where $Y=A_{2}, C_{3}(p \neq 3), B_{5}$ and $F_{4}(p \neq 3)$. If $Y=B_{5}$ then $Y<D_{6}<G$, so $Y$ is non-maximal. It remains to show that the other cases do not give factorizations.

Suppose $Y=A_{2}$. If $p \neq 3$ then $G=S_{8}$ and there is a maximal torus of $Y$ fixing pointwise a non-degenerate 2-space, hence contained in $Y \cap N_{1}$, so $\operatorname{dim} Y=$ $8<\operatorname{dim}\left(G / N_{1}\right)+\operatorname{dim} X \cap Y$, hence $G \neq Y N_{1}$. And if $p=3$, then $Y=A_{2}<G_{2}<$ $G=S O_{7}$, so $Y$ is non-maximal in $G$.

Now let $Y=C_{3}$ with $p \neq 3$, so $G=S O_{14}$. If $W$ is the usual 6-dimensional $Y$-module $V_{Y}\left(\lambda_{1}\right)$, then $V=V_{Y}\left(\lambda_{2}\right)$ is a submodule of codimension 1 in $\bigwedge^{2} W$. Hence the maximal rank subgroup $A_{1} A_{1} A_{1}$ of $Y$ fixes nonsingular vectors in $V$, so lies in $Y \cap N_{1}$. Therefore $G \neq Y N_{1}$ by the usual count of dimensions.

Finally, consider $Y=F_{4}$ with $p \neq 3$. Here $G=S O_{26}$. Let $D$ be a maximal rank subgroup $B_{4}$ of $Y$. Then $V \downarrow D=V_{D}\left(\lambda_{4}\right) \oplus M$, where $M$ is a $D$-submodule of dimension 10 having one composition factor $V_{D}\left(\lambda_{1}\right)$ and the other $1+\delta_{p, 2}$ factors trivial. It follows that $D$ fixes a nonsingular 1 -space in $M$, so we may take it that $D \leq Y \cap N_{1}$. Since $\operatorname{dim} Y / D<\operatorname{dim} G / N_{1}$, it follows that $G \neq Y N_{1}$.

In view of 5.2, we assume from now on that neither $X$ nor $Y$ is $N_{k}$.

Lemma 5.3. We have $X \neq S p(V)$.

Proof. Suppose $X=S p_{2 m}, G=S L_{2 m}$. If $m=2$, then $X$ corresponds to the subgroup $N_{1}$ in $S O_{6} \cong G /\langle-I\rangle$. So we assume that $m \geq 3$. We have $\operatorname{dim} Y \geq$ $\operatorname{dim}(G / X)=2 m^{2}-m-1$. This implies that $Y \neq C l(U) \otimes C l(W)$ with $V=U \otimes W$, so 2.4(iii) or 2.4(iv) holds for $Y$. Certainly $\operatorname{dim} V \leq \frac{1}{2} \operatorname{dim} Y+2$, so from 2.9 we deduce that 2.4(iii) holds - that is, $Y=C l(V)$. Since $X, Y$ are non-conjugate, it follows that $Y=S O_{2 m}$ and $p \neq 2$. Then

$$
\operatorname{dim} X \cap Y=\operatorname{dim} Y-\operatorname{dim}(G / X)=1 .
$$

However, a maximal torus in $\mathrm{SO}_{2 m}$ fixes both a non-degenerate symmetric bilinear form on $V$ and a non-degenerate skew-symmetric bilinear form on $V$, hence lies in a subgroup $S p_{2 m}$. Hence $\operatorname{dim} X \cap Y \geq m$, a contradiction.

Lemma 5.4. If $(X, G)=(S O(V), S p(V))$ with $p=2\left(\right.$ and $\left.Y \neq N_{k}\right)$, then $\operatorname{dim} V=$ 6 and $Y=G_{2}$, as in Theorem $B(4)$.

Proof. Let $\operatorname{dim} V=2 m$. Clearly $Y \not \leq S O(V)$. Since $p=2, Y$ is not $C l\left(V_{1}\right) \otimes C l\left(V_{2}\right)$ as in 2.4(ii) (all these subgroups lie in $S O(V)$ when $p=2$ ). Also $Y \neq N_{k}$, so $Y$ must 
be simple and irreducible on $V$, as in 2.4(iv). Moreover, if $T$ is a maximal torus of $Y$, then $T$ lies in a maximal torus of a subgroup $S O(V)$ of $G$ (since maximal tori of $S O(V)$ and $S p(V)$ coincide), and hence we may take it that $T \leq X \cap Y$. We may also assume that $\operatorname{rank}(Y)=k>1$. Thus

$$
\operatorname{dim} Y-k \geq \operatorname{dim}(G / X)=2 m=\operatorname{dim} V .
$$

Write $V \downarrow Y=V_{Y}(\lambda)$. Then by 2.8, either $V$ is a composition factor of the adjoint module for $Y$, or $(Y, \lambda)=\left(G_{2}, \lambda_{1}\right)$. If $(Y, \lambda)=\left(G_{2}, \lambda_{1}\right)$, the conclusion of the lemma holds.

Assume now that $V$ is a composition factor of the adjoint module for $Y$. Since $\operatorname{dim} V \leq \operatorname{dim} Y-k$ and $k \geq 2$, it must be the case that $Y=B_{n}, C_{n}, D_{n}$ or $F_{4}$. In the last case the adjoint module has two composition factors, $V_{Y}\left(\lambda_{1}\right)$ and $V_{Y}\left(\lambda_{4}\right)$, both of dimension 26; since neither of the Weyl modules $W_{Y}\left(\lambda_{1}\right), W_{Y}\left(\lambda_{4}\right)$ has a trivial composition factor, the proof of Lemma 2.6 shows that $Y \leq S O(V)$, a contradiction. Thus $Y=B_{n}, C_{n}$ or $D_{n}$. Clearly $V$ is not the usual $2 n$-dimensional module for $Y$, so $V=V_{Y}\left(\lambda_{2}\right)$. The image of $B_{n}$ in $S L(V)$ in this representation is $C_{n}$; moreover, $D_{n}<C_{n}<S p(V)$. Therefore by the maximality of $Y$ in $G, Y=C_{n}$.

We conclude that $Y=C_{n}$ and $V=V_{Y}\left(\lambda_{2}\right)$. If $n$ is odd, then $V_{Y}\left(\lambda_{2}\right)=W_{Y}\left(\lambda_{2}\right)$, so 2.6 gives $Y<S O(V)$, a contradiction. Therefore $n$ is even.

If $n=2$, then $\operatorname{dim} V=4$ and $Y=G$; so $n \geq 4$. Let $W$ be the usual $2 n$ dimensional $Y$-module $V_{Y}\left(\lambda_{1}\right)$. Then there is a series of $Y$-submodules

$$
0<V_{1}<V_{2}<\bigwedge^{2} W
$$

with $\operatorname{dim} V_{1}=\operatorname{dim}\left(\bigwedge^{2} W / V_{2}\right)=1$, such that $V=V_{Y}\left(\lambda_{2}\right)=V_{2} / V_{1}$; in particular, $\operatorname{dim} V=2 m=n(2 n-1)-2$. Choose a maximal rank subgroup $C D=C_{1} C_{n-1}$ in $Y$. Then

$$
V \downarrow C D=V_{D}\left(\lambda_{2}\right) \oplus\left(V_{C}\left(\lambda_{1}\right) \otimes V_{D}\left(\lambda_{1}\right)\right) .
$$

By 2.8 , since $n-1$ is odd, $D$ preserves a quadratic form on $V_{D}\left(\lambda_{2}\right)$; and $C D$ preserves a quadratic form on $V_{C}\left(\lambda_{1}\right) \otimes V_{D}\left(\lambda_{1}\right)$. Hence $C D$ lies in a subgroup $S O(V)$ of $G$, so we may take it that $C D \leq X \cap Y$. But then

$$
\operatorname{dim} Y-\operatorname{dim} X \cap Y \leq \operatorname{dim} C_{n}-\operatorname{dim} C_{1} C_{n-1}=4 n-4<\operatorname{dim} V=\operatorname{dim}(G / X),
$$

and so $G \neq X Y$.

The final lemma deals with the last remaining possibilities for $X$ given by 5.1.

Lemma 5.5. If $(X, G)=\left(B_{3}, S O_{8}\right),\left(G_{2}, S O_{7}\right)(p \neq 2)$ or $\left(G_{2}, S p_{6}\right)(p=2)$, then $G, X, Y$ are as in Theorem $B$.

Proof. If $X=B_{3}$, then there is a triality automorphism $\tau$ of $G$ such that $X^{\tau}=$ $N_{1}$. The factorization $G=X^{\tau} Y^{\tau}$ is then given by 5.2 ; hence $G=X Y$ is as in Theorem B. Now suppose $X=G_{2}$. Then $\operatorname{dim} Y \geq \operatorname{dim}(G / X)=7$. We are assuming $Y \neq N_{k}$; and if $Y=S O_{6}(p=2)$, the result follows from 5.4. So we assume that $Y \neq N_{k}, S O_{6}$. Then by 2.4 and 2.5 , we have $p=3$ and $Y=A_{2}$ or $G_{2}$ (with $V \downarrow Y=V_{Y}\left(\lambda_{1}+\lambda_{2}\right)$ or $V_{Y}\left(\lambda_{1}\right)$, respectively). But then $X$ contains a conjugate of $Y$, which means that $G \neq X Y$.

Lemmas 5.1-5.5 complete the proof of Theorem B. 


\section{APPENDIX}

In this Appendix we use Theorem B to determine all factorizations $G=X Y$ with $G$ a classical group, $X, Y$ closed proper subgroups, and $X, Y$ either reductive or parabolic. We do this by finding the minimal factorizations, where a factorization $G=X Y$ is minimal if $G \neq X_{0} Y_{0}$ for all closed subgroups $X_{0}, Y_{0}$ of $X, Y$, at least one of which is proper.

Theorem C. Let $G$ be a classical simple algebraic group in characteristic $p$. The minimal factorizations $G=X Y$ of $G$ as a product of closed proper subgroups $X, Y$, each of which is either reductive or parabolic, are as follows:

(1) generic factorizations:

$$
\begin{aligned}
& S L_{2 m}=S p_{2 m} S L_{2 m-1}, \\
& S O_{2 m}=N_{1} S L_{m}(m \text { odd }), \\
& S O_{2 m}=N_{1} S p_{m}(m \text { even, }(m, p) \neq(6,2)) \\
& S O_{2 m}=N_{1}\left(S O_{m} \otimes S p_{2}\right)(m \text { even, } m \neq 8, p=2), \\
& S p_{2 m}=S O_{2 m}\left(\left(S p_{4}\right)^{t} \times\left(S p_{2}\right)^{u}\right)(p=2,2 m=8 t+2 u)
\end{aligned}
$$

(2) factorizations in bounded dimensions:

$$
\begin{aligned}
& S O_{32}=B_{5} N_{1}(p=2), \\
& S O_{16}=\left(B_{3} \otimes S p_{2}\right) N_{1}(p=2), \\
& S O_{12}=G_{2} N_{1}(p=2), \\
& S p_{8}=B_{3} N_{2}(p=2), \\
& S O_{8}=B_{3} A_{1}^{3}=B_{3} S p_{4}=B_{3} S O_{5}, \\
& S O_{7}=G_{2} S O_{5}, \\
& S O_{7}=A_{2} N_{1}(p=3), \\
& S p_{6}=G_{2}\left(S p_{2}\right)^{3}=G_{2} S p_{4}=G_{2} S O_{5}(p=2), \\
& S L_{6}=G_{2} S L_{5}(p=2) ;
\end{aligned}
$$

(3) factorizations occurring in Theorem B:

$$
\begin{aligned}
& S O_{56}=E_{7} N_{1}(p=2), \\
& S O_{25}=F_{4} N_{1}(p=3), \\
& S O_{20}=A_{5} N_{1}(p=2), \\
& S O_{16}=B_{4} N_{1}(p \neq 2), \\
& S O_{13}=C_{3} N_{1}(p=3) .
\end{aligned}
$$

Remarks. (a) In particular, classical groups have no minimal parabolic factorizations. (This is not too surprising, in view of 2.2.)

(b) The embeddings of a few of the subgroups in the list require some elucidation:

(i) In the last case of (1), the subgroup $\left(S p_{4}\right)^{t} \times\left(S p_{2}\right)^{u}$ of $S p_{2 m}$ (with $2 m=$ $8 t+2 u)$ lies in $\left(S p_{4} \times S p_{4}\right)^{t} \times\left(S p_{2}\right)^{u}$, with $S p_{4}$ embedded in $S p_{4} \times S p_{4}$ as $\left\{\left(g, g^{\tau}\right)\right.$ : $\left.g \in S p_{4}\right\}$, where $\tau$ involves a graph automorphism of $S p_{4}$.

(ii) In the third case of (2), the subgroup $G_{2}<S p_{6}<S p_{6} \otimes S p_{2}<S O_{12}$.

(iii) In the fifth case of (2), $A_{1}^{3}$ is either $S O_{4} \otimes S p_{2}<S p_{4} \otimes S p_{2}<S O_{8}$, or $\mathrm{SO}_{4} \times \mathrm{SO}_{3}<\mathrm{N}_{3}<\mathrm{SO}_{8}$.

(iv) In the seventh case of (2), the factor $A_{2}$ is irreducible on the natural 7dimensional module (which is a composition factor of the adjoint module for $A_{2}$ ).

Sketch proof of Theorem C. By 1.1 and minimality, $X$ and $Y$ are connected. Choose maximal connected subgroups $X_{1}, Y_{1}$ of $G$ containing $X, Y$ respectively. 
Then the factorization $G=X_{1} Y_{1}$ is in the list in Theorem B. Moreover, we have

$$
X_{1}=X\left(X_{1} \cap Y\right), \quad Y_{1}=\left(X \cap Y_{1}\right) Y .
$$

Using this, it is usually a simple matter to determine the "sub-factorizations" of a given maximal factorization $G=X_{1} Y_{1}$ in Theorem B. We do this for the factorizations in Theorem $\mathrm{B}$ of large dimension:

$$
S L_{2 m}=S p_{2 m} P_{1}, S O_{2 m}=N_{1} P_{m}, S O_{2 m}=\left(S p_{m} \otimes S p_{2}\right) N_{1}, S p_{2 m}=S O_{2 m} N_{2 k},
$$

and leave the rest to the reader.

Suppose first that $G=X_{1} Y_{1}$ is $S L_{2 m}=S p_{2 m} P_{1}$. Write $Q=R_{u}\left(Y_{1}\right)$ and let $T_{1}$ be the centre of a Levi subgroup of $Y_{1}$. If $Y<Y_{1}$ then $S L_{2 m}=S p_{2 m} Y$. When $Y$ is parabolic, Theorem B implies that $Y$ lies in the intersection of $P_{1}$ and $P_{2 m-1}$; but then it is easy to see that $S L_{2 m} \neq S p_{2 m} Y$. Thus $Y$ is reductive, and writing $\bar{Y}$ for the image of $Y$ modulo $Q T_{1}$, we have $S L_{2 m-1}=S p_{2 m-2} \bar{Y}$, whence $\bar{Y}=S L_{2 m-1}$ by Theorem B. In fact, $S L_{2 m}=S p_{2 m} S L_{2 m-1}$, since the subgroup $S L_{2 m-1}$ is the stabilizer of a vector $v$ and a hyperplane $H$ not containing $v$, and $S p_{2 m}$ is transitive on such pairs $v, H$. If $X<X_{1}$, then $S p_{2 m}=X P_{1}$; hence by Theorem B, we have $p=2, m=3$ and this factorization is $S p_{6}=G_{2} P_{1}$. In fact $S p_{6}=G_{2} S p_{4}$ : for $G_{2}$ is transitive on the set of non-zero vectors, so $S p_{6}=G_{2} P_{1}^{\prime}$, and the assertion now follows from the proof of 2.2. Hence $S L_{6}=S p_{6} S L_{5}=G_{2} S p_{4} S L_{5}=G_{2} S L_{5}$. We have now established that the minimal sub-factorizations of $S L_{2 m}=S p_{2 m} P_{1}$ are

$$
S L_{2 m}=S p_{2 m} S L_{2 m-1}((m, p) \neq(3,2)) \text { and } S L_{6}=G_{2} S L_{5}(p=2) .
$$

In both cases the conjugacy class of $Y$ in $G$ is uniquely determined, as can be seen using [LS2, 1.5].

Now suppose $G=X_{1} Y_{1}$ is $S O_{2 m}=N_{1} P_{m}(m \geq 4)$. If $X<X_{1}$, then $S O_{2 m-1}=$ $X P_{m-1}$; there is no such factorization by Theorem B. Assume then that $Y<Y_{1}$. If $Y$ is parabolic, then Theorem B implies that $Y \leq P_{m} \cap P_{m-1}$, which is the stabilizer of a totally singular $(m-1)$-space, $W$ say. But there are nonsingular 1-spaces inside and outside $W^{\perp}$, so $G \neq N_{1} Y$. Therefore $Y$ is reductive. Let $\bar{Y}$ be the image of $Y$ modulo $Q T_{1}$ (defined as in the previous paragraph). Then $S L_{m}=S L_{m-1} \bar{Y}$. By the previous paragraph, $\bar{Y}$ is $S L_{m}, S p_{m}$ ( $m$ even) or $G_{2}(m=6, p=2)$. Hence we obtain the minimal factorizations

$$
\begin{gathered}
S O_{2 m}=N_{1} S L_{m}(m \text { odd }), S O_{2 m}=N_{1} S p_{m}(m \text { even, }(m, p) \neq(6,2)), \\
S O_{12}=N_{1} G_{2}(p=2) .
\end{gathered}
$$

The existence of the first two of these is given by the proofs of 1.2 and 1.3; for the existence of the last one, use $S O_{12}=N_{1} S p_{6}$ and $S p_{6}=G_{2} S p_{4}$, noting that the factor $S p_{4}$ lies in a subgroup $N_{1}$ of $S O_{12}$. As above, the conjugacy class of $Y$ in $G$ can be seen to be uniquely determined, using [LS2, 1.5].

Next suppose $G=X_{1} Y_{1}$ is $S O_{2 m}=\left(S p_{m} \otimes S p_{2}\right) N_{1}(m$ even, $m \geq 4)$. Here $X_{1} \cap Y_{1}=S p_{m-2} \times S p_{2}$, where the factor $S p_{2}$ is a diagonal subgroup of a subgroup $S p_{2} \times S p_{2}$ in $X_{1}$. If $X<X_{1}$, then $S p_{m} \otimes S p_{2}=X\left(X_{1} \cap Y_{1}\right)$ implies that $S p_{m}=$ $X_{0} N_{2}$, where $X_{0}$ is the projection of $X$ in $S p_{m}$. Hence by Theorem B, either $X_{0}=S p_{m}$ or $X_{0} \leq S O_{m}(p=2)$ or $m=6, X_{0}=G_{2}(p=2)$. In the case where $X_{0} \leq S O_{m}$ we have $S O_{m}=X_{0} N_{2}=X_{0} P_{1}$, whence either $X_{0}=S O_{m}$ or $m=8$ and $X_{0}=B_{3}$.

If $Y<Y_{1}$, then $S O_{2 m-1}=Y\left(X_{1} \cap Y_{1}\right)$. When $p \neq 2$ this gives $S O_{2 m-1}=Y N_{3}$, which is not possible by Theorem B. And when $p=2$, applying a morphism 
$S O_{2 m-1} \rightarrow S p_{2 m-2}$ we have $S p_{2 m-2}=Y N_{2}$ which forces either $Y \leq S O_{2 m-2}$ or $m=4, Y=G_{2}$. In the first case,

$$
S p_{2 m-2}=S O_{2 m-2}\left(\left(S p_{m-2} \otimes S p_{2}\right) \times S p_{2}\right),
$$

which is not possible as the factor $S p_{m-2} \otimes S p_{2}$ lies in $S O_{2 m-2}$. The second case is also impossible as $S p_{6} \neq G_{2}\left(S p_{2} \times S p_{2}\right)$ by dimension considerations.

We conclude that the minimal factorizations in this case $\left(X_{1} Y_{1}=\right.$ $\left.\left(S p_{m} \otimes S p_{2}\right) N_{1}\right)$ are

$$
\begin{gathered}
S O_{2 m}=S p_{m} N_{1}((m, p) \neq(6,2)), S O_{2 m}=\left(S O_{m} \otimes S p_{2}\right) N_{1}(p=2, m \neq 8), \\
S O_{16}=\left(B_{3} \otimes S p_{2}\right) N_{1}(p=2), S O_{12}=G_{2} N_{1}(p=2) .
\end{gathered}
$$

Finally, consider the case where $G=X_{1} Y_{1}$ is $S p_{2 m}=S O_{2 m} N_{2 k}(p=2)$. If $X<X_{1}$, then $S O_{2 m}=X N_{2 k}$, whence by Theorem B, $m=4, X=B_{3}$ and we have the factorization $S p_{8}=B_{3} N_{2}$; one checks that this factorization is minimal. Now suppose $Y<Y_{1}$. Let $Y^{(1)}, Y^{(2)}$ be the projections of $Y$ in the factors $S p_{2 k}, S p_{2 m-2 k}$ of $Y_{1}=N_{2 k}$, respectively. Then $S p_{2 k}=S O_{2 k} Y^{(1)}$ and $S p_{2 m-2 k}=S O_{2 m-2 k} Y^{(2)}$. If $Y^{(1)}=S p_{2 k}$ and $Y^{(2)}=S p_{2 m-2 k}$, then $2 k=m$ and $Y \cong S p_{m}$ is a diagonal subgroup of $Y_{1}=S p_{m} \times S p_{m}$, say $Y=\left\{\left(a, a^{\tau}\right): a \in S p_{m}\right\}$, where $\tau$ is an automorphism of $S p_{m}$ (as abstract group). This implies that $S p_{m}=\left(S O_{m}\right)\left(S O_{m}\right)^{\tau}$, whence $m=4$ and $\tau$ involves a graph automorphism of $S p_{4}$. If $Y^{(1)}<S p_{2 k}$, then by Theorem B, $Y^{(1)} \leq N_{2 l}$ for some $l<k$, and we repeat the above argument. Hence we obtain the minimal factorizations

$$
S p_{8}=B_{3} N_{2}, \quad S p_{2 m}=S O_{2 m}\left(S p_{4}^{t} \times S p_{2}^{u}\right)(\text { with } 2 m=8 t+2 u) .
$$

\section{REFERENCES}

[Bo] A. Borel, Linear algebraic groups, 2nd ed., Springer-Verlag, New York, 1991. MR 92d:20001

[Bou] N. Bourbaki, Groupes et algèbres de Lie, Chapter 4, Hermann, Paris, 1968. MR 39:1590

[Ca] R. W. Carter, Conjugacy classes in the Weyl group, Compositio Math. 25 (1972), 1-59. MR 47:6884

[CLSS] A. M. Cohen, M.W. Liebeck, J. Saxl, and G. M. Seitz, The local maximal subgroups of exceptional groups of Lie type, finite and algebraic, Proc. London Math. Soc. 64 (1992), 21-48. MR 92m:20012

[Ha] W. J. Haboush, Homogeneous vector bundles and reductive subgroups of reductive algebraic groups, Amer. J. Math. 100 (1978), 1123-1137. MR 80f:14007

$[\mathrm{Hu}]$ J. E. Humphreys, Linear algebraic groups, Graduate Texts in Math., No. 21, Springer, 1975. MR 53:633

[Ka] I. L. Kantor, Cross-ratio of four points and other invariants on homogeneous spaces with parabolic isotropy groups, Trudy Sem. Vektor. Tenzor. Anal. 17 (1974), 250-313. MR 50:7176

[KST] P. B. Kleidman, G.M. Seitz and D.M. Testerman, preprint.

[Li] M. W. Liebeck, The affine permutation groups of rank three, Proc. London Math. Soc. 54 (1987), 477-516. MR 88m:20004

[LPS] M. W. Liebeck, C.E. Praeger, and J. Saxl, The maximal factorizations of the finite simple groups and their automorphism groups, Mem. Amer. Math. Soc., Vol. 86, No. 432 (1990), 1-151. MR 90k:20048

[LS1] M. W. Liebeck and G.M. Seitz, Subgroups containing root elements in groups of Lie type, Annals of Math. (2) 139 (1994), 293-361. MR 95d:20078

[LS2] — Reductive subgroups of exceptional algebraic groups, Mem. Amer. Math. Soc. (to appear).

[On] A. L. Onishchik, Parabolic factorizations of semisimple algebraic groups, Math. Nachr. 104 (1981), 315-329. MR 83h:20041 
[Ri] R. W. Richardson, Affine coset spaces of reductive algebraic groups, Bull. London Math. Soc. 9 (1977), 38-41. MR 55:10473

[Se1] G. M. Seitz, The maximal subgroups of classical algebraic groups, Mem. Amer. Math. Soc., Vol. 67, No. 365 (1987), 1-286. MR 88g:20092

[Se2] - The maximal subgroups of exceptional algebraic groups, Mem. Amer. Math. Soc., Vol. 90, No. 441 (1991), 1-197. MR 91g:20038

[SS] T. A. Springer and R. Steinberg, Conjugacy classes, Seminar on Algebraic Groups and Related Topics (A. Borel et al., eds.), Lecture Notes in Math., vol. 131, Springer, Berlin, 1970, pp. 168-266. MR 42:3091

[St1] R. Steinberg, Lectures on Chevalley groups, Yale University Lecture Notes, 1968.

[St2] Conjugacy classes in algebraic groups, Lecture Notes in Math., vol. 366, Springer, Berlin, 1974. MR 50:4766

Department of Mathematics, Imperial College, London SW7 2BZ, England

E-mail address: m.liebeck@ic.ac.uk

Department of Pure Mathematics and Mathematical Statistics, University of CamBridge, 16 Mill Lane, Cambridge CB2 1SB, England

E-mail address: j.saxl@pmms.cam.ac.uk

Department of Mathematics, University of Oregon, Eugene, Oregon 97403

E-mail address: seitz@bright.uoregon.edu 